\title{
Orthogonal Gyroexpansion in Möbius Gyrovector Spaces
}

\author{
Keiichi Watanabe \\ Department of Mathematics, Faculty of Science, Niigata University, Niigata 950-2181, Japan \\ Correspondence should be addressed to Keiichi Watanabe; wtnbk@math.sc.niigata-u.ac.jp
}

Received 12 August 2017; Accepted 17 September 2017; Published 13 December 2017

Academic Editor: Juan Martinez-Moreno

Copyright (C) 2017 Keiichi Watanabe. This is an open access article distributed under the Creative Commons Attribution License, which permits unrestricted use, distribution, and reproduction in any medium, provided the original work is properly cited.

\begin{abstract}
We investigate the Möbius gyrovector spaces which are open balls centered at the origin in a real Hilbert space with the Möbius addition, the Möbius scalar multiplication, and the Poincaré metric introduced by Ungar. In particular, for an arbitrary point, we can easily obtain the unique closest point in any closed gyrovector subspace, by using the ordinary orthogonal decomposition. Further, we show that each element has the orthogonal gyroexpansion with respect to any orthogonal basis in a Möbius gyrovector space, which is similar to each element in a Hilbert space having the orthogonal expansion with respect to any orthonormal basis. Moreover, we present a concrete procedure to calculate the gyrocoefficients of the orthogonal gyroexpansion.
\end{abstract}

\section{Introduction}

A. Ungar initiated study on gyrogroups and gyrovector spaces (cf. [1]). Gyrovector spaces are generalized vector spaces, with which they share important analogies, just as gyrogroups are analogous to groups. The first known gyrogroup was the ball of Euclidean space $\mathbb{R}^{3}$ endowed with Einstein's velocity addition associated with the special theory of relativity. Another example of a gyrogroup is the open unit disc in the complex plain endowed with the Möbius addition. Ungar extended these gyroadditions to the ball of an arbitrary real inner product space, introduced a common gyroscalar multiplication, and observed that the ball endowed with gyrooperations are gyrovector spaces (cf. [2, 3]). He describes that gyrovector spaces provide the setting for hyperbolic geometry just as vector spaces provide the setting for Euclidean geometry. In particular, Möbius gyrovector spaces form the setting for the Poincaré ball model of hyperbolic geometry, and similarly, Einstein gyrovector spaces form the setting for the Beltrami-Klein ball model. Readers may consult $[4,5]$ and the references therein for general information about gyrogroups and gyrovector spaces.

Gyrooperations are generally not commutative, associative, or distributive. Thus the theory of gyrovector spaces falls within the general area of nonlinear functional analysis. They are enjoying algebraic rules such as left and right gyroassociative, gyrocommutative, scalar distributive, and scalar associative laws, so there exist rich symmetrical structures which we should clarify precisely. Many elementary problems are still unsolved. We refer to [6-10] as examples of recent papers for gyrovector spaces, their generalizations, and related matters.

In [8], Abe and the author of the present article showed that any finitely generated gyrovector subspace in the Möbius gyrovector space coincides with the intersection of the linear subspace generated by the same generators and the Möbius ball. As an application, they presented a notion of orthogonal gyrodecomposition and clarified the relation to the ordinary orthogonal decomposition.

The importance of the orthogonal expansion of each vector with respect to an orthonormal basis in a Hilbert space cannot be overemphasized in both theory and application of functional analysis. In this paper we will introduce a concept of orthogonal gyroexpansion of each element with respect to an orthogonal basis in a Möbius gyrovector space and reveal analogies that it shares with its classical counterpart. Such problems seem to be quite fundamental and important for developing pure and applied mathematics, since one of the virtues of gyrovector spaces is that they have properties which are fully analogous to vector space properties. Moreover, the gyrocoefficients of the orthogonal gyroexpansion can be concretely calculated by a procedure that is given here.

The paper is organized as follows. Section 2 is the preliminaries. In Section 3, we introduce a notion of gyrolinear 
independency for finite sets in a gyrovector space and show that it coincides with the notion of the linear independency. In Section 4, we give a notion of orthogonal gyroexpansions with respect to a complete orthogonal sequence in the Möbius gyrovector space, and we present an explicit procedure to obtain the orthogonal gyroexpansions.

\section{Preliminaries}

Let us briefly recall the definitions of two models of gyrovector spaces, that is, the Möbius and Einstein gyrovector spaces. For precise definitions and basic results of gyrocommutative gyrogroups and gyrovector spaces, see [4].

Let $\mathbb{V}=(\mathbb{V},+, \cdot)$ be a real inner product space with a binary operation + and a positive definite inner product $\cdot$ and let $\mathbb{V}_{s}$ be the ball

$$
\mathbb{V}_{s}=\{\mathbf{a} \in \mathbb{V}:\|\mathbf{a}\|<s\}
$$

for any fixed $s>0$.

Definition 1 (see [4, Definitions 3.40 and 6.83]). The Möbius addition $\oplus_{M}$ and the Möbius scalar multiplication $\otimes_{M}$ are given by the equations

$$
\begin{aligned}
& \mathbf{a} \oplus_{M} \mathbf{b} \\
& =\frac{\left(1+\left(2 / s^{2}\right) \mathbf{a} \cdot \mathbf{b}+\left(1 / s^{2}\right)\|\mathbf{b}\|^{2}\right) \mathbf{a}+\left(1-\left(1 / s^{2}\right)\|\mathbf{a}\|^{2}\right) \mathbf{b}}{1+\left(2 / s^{2}\right) \mathbf{a} \cdot \mathbf{b}+\left(1 / s^{4}\right)\|\mathbf{a}\|^{2}\|\mathbf{b}\|^{2}} \\
& r \otimes_{M} \mathbf{a}=s \tanh \left(r \tanh ^{-1} \frac{\|\mathbf{a}\|}{s}\right) \frac{\mathbf{a}}{\|\mathbf{a}\|}
\end{aligned}
$$

$$
\text { (if } \mathbf{a} \neq \mathbf{0}), r \otimes_{\mathrm{M}} \mathbf{0}=\mathbf{0}
$$

for any $\mathbf{a}, \mathbf{b} \in \mathbb{V}_{s}, r \in \mathbb{R}$. The addition $\oplus_{\mathrm{M}}$ and scalar multiplication $\otimes_{M}$ for the set $\left\|\mathbb{V}_{s}\right\|=\left\{ \pm\|\mathbf{a}\| ; \mathbf{a} \in \mathbb{V}_{s}\right\}$ in the axiom (VV) of gyrovector space are defined by the equations

$$
\begin{aligned}
& a \oplus_{\mathrm{M}} b=\frac{a+b}{1+\left(1 / s^{2}\right) a b} \\
& r \otimes_{\mathrm{M}} a=s \tanh \left(r \tanh ^{-1} \frac{a}{s}\right)
\end{aligned}
$$

for any $a, b \in\left\|\mathbb{V}_{s}\right\|, r \in \mathbb{R}$.

We simply denote $\oplus_{M}, \otimes_{M}$ by $\oplus, \otimes$, respectively. If several kinds of operations appear in a formula simultaneously, we always give priority by the following order: (i) ordinary scalar multiplication; (ii) gyroscalar multiplication $\otimes$; (iii) gyroaddition $\oplus$; that is,

$$
\begin{aligned}
r_{1} \otimes w_{1} \mathbf{a}_{1} \oplus r_{2} \otimes w_{2} \mathbf{a}_{2}= & \left\{r_{1} \otimes\left(w_{1} \mathbf{a}_{1}\right)\right\} \\
& \oplus\left\{r_{2} \otimes\left(w_{2} \mathbf{a}_{2}\right)\right\},
\end{aligned}
$$

and the parentheses are omitted in such cases. In general, we note that gyroaddition does not distribute with (both ordinary and gyro) scalar multiplications:

$$
\begin{gathered}
t(\mathbf{a} \oplus \mathbf{b}) \neq t \mathbf{a} \oplus t \mathbf{b}, \\
r \otimes(\mathbf{a} \oplus \mathbf{b}) \neq r \otimes \mathbf{a} \oplus r \otimes \mathbf{b} .
\end{gathered}
$$

In the limit of large $s, s \rightarrow \infty$, the ball $\mathbb{V}_{s}$ expands to the whole space $\mathbb{V}$. The next proposition suggests that each result in linear analysis can be restored from the counterpart in gyrolinear analysis.

Proposition 2 (see [4, p. 78]). The Möbius addition (resp., Möbius scalar multiplication) reduces to the vector addition (resp., scalar multiplication) as $s \rightarrow \infty$; that is,

$$
\begin{aligned}
& \mathbf{a} \oplus \mathbf{b} \longrightarrow \mathbf{a}+\mathbf{b} \quad(s \longrightarrow \infty) \\
& r \otimes \mathbf{a} \longrightarrow r \mathbf{a} \quad(s \longrightarrow \infty) .
\end{aligned}
$$

Definition 3 (see [4, Definitions 3.45 and 6.86]). The Einstein addition $\oplus_{\mathrm{E}}$ and the Einstein scalar multiplication $\otimes_{\mathrm{E}}$ are given by the equations

$$
\begin{array}{r}
\mathbf{a} \oplus_{\mathrm{E}} \mathbf{b}=\frac{1}{1+(\mathbf{a} \cdot \mathbf{b}) / s^{2}}\left\{\mathbf{a}+\frac{1}{\gamma_{\mathbf{a}}} \mathbf{b}+\frac{1}{s^{2}} \frac{\gamma_{\mathbf{a}}}{1+\gamma_{\mathbf{a}}}(\mathbf{a} \cdot \mathbf{b}) \mathbf{a}\right\} \\
r \otimes_{\mathrm{E}} \mathbf{a}=s \tanh \left(r \tanh ^{-1} \frac{\|\mathbf{a}\|}{s}\right) \frac{\mathbf{a}}{\|\mathbf{a}\|} \\
(\text { if } \mathbf{a} \neq \mathbf{0}), r \otimes_{\mathrm{E}} \mathbf{0}=\mathbf{0}
\end{array}
$$

for any $\mathbf{a}, \mathbf{b} \in \mathbb{V}_{s}, r \in \mathbb{R}$, where $\gamma_{\mathbf{a}}=1 / \sqrt{1-\|\mathbf{a}\|^{2} / s^{2}}$.

Note that each of the Einstein scalar multiplication and the operations on the set $\left\|\mathbb{V}_{s}\right\|$ is identical to the corresponding operation for the Möbius gyrovector spaces.

Definition 4 (see [4, Definition 6.88]). An isomorphism from a gyrovector space $\left(G_{1}, \oplus_{1}, \otimes_{1}\right)$ to a gyrovector space $\left(G_{2}, \oplus_{2}, \otimes_{2}\right)$ is a bijective map $\phi: G_{1} \rightarrow G_{2}$ that preserves gyrooperations and keeps the inner product of normalized elements invariant; that is,

$$
\begin{aligned}
\phi\left(\mathbf{a} \oplus_{1} \mathbf{b}\right) & =\phi(\mathbf{a}) \oplus_{2} \phi(\mathbf{b}) \\
\phi\left(r \otimes_{1} \mathbf{a}\right) & =r \otimes_{2} \phi(\mathbf{a}) \\
\frac{\phi(\mathbf{a})}{\|\phi(\mathbf{a})\|} \cdot \frac{\phi(\mathbf{b})}{\|\phi(\mathbf{b})\|} & =\frac{\mathbf{a}}{\|\mathbf{a}\|} \cdot \frac{\mathbf{b}}{\|\mathbf{b}\|} \quad(\text { if } \mathbf{a}, \mathbf{b} \neq \mathbf{0})
\end{aligned}
$$

for any $\mathbf{a}, \mathbf{b} \in G_{1}, r \in \mathbb{R}$.

Theorem 5 (see [4, Table 6.1]). Let $\phi_{\mathrm{EM}}: \mathbb{V}_{s} \rightarrow \mathbb{V}_{s}$ be the map defined by the equation

$$
\phi_{\mathrm{EM}}(\mathbf{a})=2 \otimes \mathbf{a}
$$

for any $\mathbf{a} \in \mathbb{V}_{s}$. Then $\phi_{\mathrm{EM}}$ is an isomorphism from the Möbius gyrovector space to the Einstein gyrovector space.

Thus, most of results established for the Möbius gyrovector spaces in the sequel can be transformed to corresponding results for the Einstein gyrovector spaces by the isomorphism stated above.

\section{Gyrolinear Independency}

We begin with consideration of a counterpart in a gyrovector space to the notion of linearly independent sets in a linear space. 
Definition 6. A finite subset $\left\{\mathbf{a}_{1}, \ldots, \mathbf{a}_{n}\right\} \subset \mathbb{V}_{s}$ is gyrolinearly independent if, for any permutation $\left(i_{1}, \ldots, i_{n}\right)$ of $\{1, \ldots, n\}$ and for any order of gyroaddition, the following implication holds:

$$
\begin{aligned}
r_{i_{1}} \otimes \mathbf{a}_{i_{1}} \oplus \cdots \oplus r_{i_{n}} \otimes \mathbf{a}_{i_{n}} & =\mathbf{0} \Longrightarrow \\
r_{1} & =\cdots=r_{n}=0 .
\end{aligned}
$$

Example 7. Let $\mathbb{V}=\mathbb{R}^{2}$ with the Euclidean inner product and $s=1$. If we identify $\mathbb{V}_{1}$ with the open unit disc $\mathbb{D}=\{z \in$ $\mathbb{C} ;|z|<1\}$ in the complex plain $\mathbb{C}$ by $(x, y)=x+i y$, then it is well-known that the Möbius addition reduces to

$$
a \oplus b=\frac{a+b}{1+\bar{a} b}
$$

for any $a, b \in \mathbb{D}($ cf. $[4,(3.127)])$. If we take

$$
\begin{aligned}
& a=\frac{i}{2}, \\
& b=-\frac{2}{5}-\frac{2}{5} i, \\
& c=\frac{1}{2}
\end{aligned}
$$

then

$$
\begin{aligned}
& a \oplus(b \oplus c)=0, \\
& (a \oplus b) \oplus c=\frac{4+16 i}{53-8 i} .
\end{aligned}
$$

This means that $\{a, b, c\}$ is not gyrolinearly independent, and if we put

$$
\begin{aligned}
& r_{1}=\frac{\tanh ^{-1}((-33+\sqrt{689}) / 20)}{\tanh ^{-1}(1 / 2)}, \\
& r_{2}=\frac{\tanh ^{-1}((17-\sqrt{689}) / 20)}{\tanh ^{-1}(1 / 2)},
\end{aligned}
$$

then it is readily checked that

$$
\begin{aligned}
r_{1} & \otimes c \oplus r_{2} \otimes a=\frac{-33+\sqrt{689}}{20} \oplus \frac{17-\sqrt{689}}{20} i \\
& =\frac{((-33+\sqrt{689}) / 20)+((17-\sqrt{689}) / 20) i}{1+((-33+\sqrt{689}) / 20) \cdot((17-\sqrt{689}) / 20) i} \\
& =b .
\end{aligned}
$$

It is immediate to see the following lemma by the fact that $1 \otimes \mathbf{a}=\mathbf{a}$ and $0 \otimes \mathbf{a}=\mathbf{0}$ and Definition 6 . We omit the proof.

Lemma 8. Let $\left\{\mathbf{a}_{1}, \ldots, \mathbf{a}_{n}\right\} \subset \mathbb{V}_{s}$ be gyrolinearly independent. Then

(i) each element is nonzero;

(ii) any subset is also gyrolinearly independent.
Lemma 9. Suppose that $\left\{\mathbf{a}_{1}, \mathbf{a}_{2}\right\}$ is linearly independent in $\mathbb{V}_{s}$ and

$$
r_{1} \otimes \mathbf{a}_{1} \oplus r_{2} \otimes \mathbf{a}_{2}=\lambda_{1} \otimes \mathbf{a}_{1} \oplus \lambda_{2} \otimes \mathbf{a}_{2}
$$

Then one has $r_{1}=\lambda_{1}$ and $r_{2}=\lambda_{2}$.

Proof. Without loss of generality, we may assume that $s=1$, $r_{j} \neq 0$, and $\lambda_{j} \neq 0$. If we put

$$
\begin{aligned}
\alpha & =\frac{\mathbf{a}_{1}}{\left\|\mathbf{a}_{1}\right\|} \cdot \frac{\mathbf{a}_{2}}{\left\|\mathbf{a}_{2}\right\|}, \\
c_{j} & =\tanh \left(r_{j} \tanh ^{-1}\left\|\mathbf{a}_{j}\right\|\right), \\
d_{j} & =\tanh \left(\lambda_{j} \tanh ^{-1}\left\|\mathbf{a}_{j}\right\|\right)
\end{aligned}
$$

for $j=1,2$, then, from the definitions of $\oplus, \otimes$, it follows that $-1 \leq \alpha \leq 1,0<\left|c_{j}\right|,\left|d_{j}\right|<1$, and

$$
\begin{aligned}
r_{1} \otimes \mathbf{a}_{1} \oplus r_{2} \otimes \mathbf{a}_{2} & =\lambda_{1} \otimes \mathbf{a}_{1} \oplus \lambda_{2} \otimes \mathbf{a}_{2} \\
& =t_{1} \frac{\mathbf{a}_{1}}{\left\|\mathbf{a}_{1}\right\|}+t_{2} \frac{\mathbf{a}_{2}}{\left\|\mathbf{a}_{2}\right\|},
\end{aligned}
$$

where we put

$$
\begin{aligned}
& t_{1}=\frac{\left(1+2 c_{1} c_{2} \alpha+c_{2}^{2}\right) c_{1}}{1+2 c_{1} c_{2} \alpha+c_{1}{ }^{2} c_{2}^{2}}=\frac{\left(1+2 d_{1} d_{2} \alpha+d_{2}^{2}\right) d_{1}}{1+2 d_{1} d_{2} \alpha+d_{1}^{2} d_{2}^{2}} \\
& t_{2}=\frac{\left(1-c_{1}^{2}\right) c_{2}}{1+2 c_{1} c_{2} \alpha+c_{1}{ }^{2} c_{2}{ }^{2}}=\frac{\left(1-d_{1}{ }^{2}\right) d_{2}}{1+2 d_{1} d_{2} \alpha+d_{1}{ }^{2} d_{2}{ }^{2}} .
\end{aligned}
$$

This means that $\left(c_{1}, c_{2}\right)$ and $\left(d_{1}, d_{2}\right)$ are solutions to the system of equations

$$
\begin{aligned}
& x^{2} y^{2}+\left(\gamma x^{2}+2 \alpha x-\gamma\right) y+1=0 \\
& x y^{2}+\left((2 \alpha+\beta) x^{2}-\beta\right) y+x=0
\end{aligned}
$$

where we put $\beta=t_{1} / t_{2}$ and $\gamma=1 / t_{2}$. Then, we have $\beta \neq 0$ and $1+\beta(2 \alpha+\beta)<\gamma^{2}$ by [8, Lemma 2.2]. So we can apply [8, Theorem 2.4] to obtain that $c_{j}=d_{j}$, which yields that $r_{j}=\lambda_{j}$ for $j=1,2$. This completes the proof.

Theorem 10. Let $\left\{\mathbf{a}_{1}, \ldots, \mathbf{a}_{n}\right\}$ be a linearly independent set in $\mathbb{V}_{s}$. Suppose that two gyrolinear combinations $r_{1} \otimes \mathbf{a}_{1} \oplus \cdots \oplus$ $r_{n} \otimes \mathbf{a}_{n}, \lambda_{1} \otimes \mathbf{a}_{1} \oplus \cdots \oplus \lambda_{n} \otimes \mathbf{a}_{n}$ are given the same order of gyroaddition and

$$
r_{1} \otimes \mathbf{a}_{1} \oplus \cdots \oplus r_{n} \otimes \mathbf{a}_{n}=\lambda_{1} \otimes \mathbf{a}_{1} \oplus \cdots \oplus \lambda_{n} \otimes \mathbf{a}_{n}
$$

Then one has $r_{j}=\lambda_{j}(j=1, \ldots, n)$.

Proof. Without loss of generality, we may assume that $s=1$. Assume that the theorem is valid up to $n$. Let $\left\{\mathbf{a}_{1}, \ldots, \mathbf{a}_{n+1}\right\}$ be a linearly independent set in $\mathbb{V}_{1}$ and let the following formula

$$
\begin{aligned}
\left(r_{1} \otimes \mathbf{a}_{1} \oplus \cdots \oplus r_{m} \otimes \mathbf{a}_{m}\right) \\
\\
\oplus\left(r_{m+1} \otimes \mathbf{a}_{m+1} \oplus \cdots \oplus r_{n+1} \otimes \mathbf{a}_{n+1}\right) \\
=\left(\lambda_{1} \otimes \mathbf{a}_{1} \oplus \cdots \oplus \lambda_{m} \otimes \mathbf{a}_{m}\right) \\
\quad \oplus\left(\lambda_{m+1} \otimes \mathbf{a}_{m+1} \oplus \cdots \oplus \lambda_{n+1} \otimes \mathbf{a}_{n+1}\right)
\end{aligned}
$$


show the latest gyroadditions. Put

$$
\begin{aligned}
\mathbf{a} & =r_{1} \otimes \mathbf{a}_{1} \oplus \cdots \oplus r_{m} \otimes \mathbf{a}_{m} \\
\mathbf{b} & =r_{m+1} \otimes \mathbf{a}_{m+1} \oplus \cdots \oplus r_{n+1} \otimes \mathbf{a}_{n+1} \\
\mathbf{a}^{\prime} & =\lambda_{1} \otimes \mathbf{a}_{1} \oplus \cdots \oplus \lambda_{m} \otimes \mathbf{a}_{m} \\
\mathbf{b}^{\prime} & =\lambda_{m+1} \otimes \mathbf{a}_{m+1} \oplus \cdots \oplus \lambda_{n+1} \otimes \mathbf{a}_{n+1} .
\end{aligned}
$$

Then $\mathbf{a}, \mathbf{a}^{\prime}$ (resp., $\mathbf{b}, \mathbf{b}^{\prime}$ ) belong to the linear span of $\left\{\mathbf{a}_{1}, \ldots, \mathbf{a}_{m}\right\}$ (resp., $\left\{\mathbf{a}_{m+1}, \ldots, \mathbf{a}_{n+1}\right\}$ ). If $\mathbf{a}=\mathbf{0}$, then we have $\mathbf{b}=\mathbf{a}^{\prime} \oplus \mathbf{b}^{\prime}$. By [8, Theorem 3.3], we can express $\mathbf{b}, \mathbf{a}^{\prime}, \mathbf{b}^{\prime}$ of the form

$$
\begin{aligned}
& \mathbf{b}=t_{m+1} \frac{\mathbf{a}_{m+1}}{\left\|\mathbf{a}_{m+1}\right\|}+\cdots+t_{n+1} \frac{\mathbf{a}_{n+1}}{\left\|\mathbf{a}_{n+1}\right\|} \\
& \mathbf{a}^{\prime}=t_{1}^{\prime} \frac{\mathbf{a}_{1}}{\left\|\mathbf{a}_{1}\right\|}+\cdots+t_{m}^{\prime} \frac{\mathbf{a}_{m}}{\left\|\mathbf{a}_{m}\right\|} \\
& \mathbf{b}^{\prime}=t_{m+1}^{\prime} \frac{\mathbf{a}_{m+1}}{\left\|\mathbf{a}_{m+1}\right\|}+\cdots+t_{n+1}^{\prime} \frac{\mathbf{a}_{n+1}}{\left\|\mathbf{a}_{n+1}\right\|} .
\end{aligned}
$$

By the definition of $\oplus$, it follows that

$$
\begin{array}{r}
t_{m+1} \frac{\mathbf{a}_{m+1}}{\left\|\mathbf{a}_{m+1}\right\|}+\cdots+t_{n+1} \frac{\mathbf{a}_{n+1}}{\left\|\mathbf{a}_{n+1}\right\|} \\
\quad=c_{1} \frac{\mathbf{a}_{1}}{\left\|\mathbf{a}_{1}\right\|}+\cdots+c_{n+1} \frac{\mathbf{a}_{n+1}}{\left\|\mathbf{a}_{n+1}\right\|},
\end{array}
$$

where we put

$$
\begin{aligned}
& c_{j} \\
& = \begin{cases}\frac{\left(1+2 \mathbf{a}^{\prime} \cdot \mathbf{b}^{\prime}+\left\|\mathbf{b}^{\prime}\right\|^{2}\right) t_{j}^{\prime}}{1+2 \mathbf{a}^{\prime} \cdot \mathbf{b}^{\prime}+\left\|\mathbf{a}^{\prime}\right\|^{2}\left\|\mathbf{b}^{\prime}\right\|^{2}} & (j=1, \ldots, m) \\
\frac{\left(1-\left\|\mathbf{a}^{\prime}\right\|^{2}\right) t_{j}^{\prime}}{1+2 \mathbf{a}^{\prime} \cdot \mathbf{b}^{\prime}+\left\|\mathbf{a}^{\prime}\right\|^{2}\left\|\mathbf{b}^{\prime}\right\|^{2}} & (j=m+1, \ldots, n+1) .\end{cases}
\end{aligned}
$$

Since $\left\{\mathbf{a}_{1}, \ldots, \mathbf{a}_{n+1}\right\}$ is linearly independent, we have $c_{1}=\cdots=$ $c_{m}=0$, which implies that $t_{1}^{\prime}=\cdots=t_{m}^{\prime}=0$; that is, $\mathbf{a}^{\prime}=\mathbf{0}$. By the assumption of our induction, it follows that $r_{j}=\lambda_{j}$ for all $j$.

Similarly, we may assume that $\mathbf{a}, \mathbf{a}^{\prime}, \mathbf{b}, \mathbf{b}^{\prime} \neq \mathbf{0}$, so $\{\mathbf{a}, \mathbf{b}\}$ is linearly independent. By the definition of $\oplus$, we can rewrite the equation

$$
\mathbf{a} \oplus \mathbf{b}=\mathbf{a}^{\prime} \oplus \mathbf{b}^{\prime}
$$

as

$$
t_{1} \frac{\mathbf{a}}{\|\mathbf{a}\|}+t_{2} \frac{\mathbf{b}}{\|\mathbf{b}\|}=t_{1}^{\prime} \frac{\mathbf{a}^{\prime}}{\left\|\mathbf{a}^{\prime}\right\|}+t_{2}^{\prime} \frac{\mathbf{b}^{\prime}}{\left\|\mathbf{b}^{\prime}\right\|},
$$

so we obtain that

$$
\begin{aligned}
& t_{1} \frac{\mathbf{a}}{\|\mathbf{a}\|}=t_{1}^{\prime} \frac{\mathbf{a}^{\prime}}{\left\|\mathbf{a}^{\prime}\right\|}, \\
& t_{2} \frac{\mathbf{b}}{\|\mathbf{b}\|}=t_{2}^{\prime} \frac{\mathbf{b}^{\prime}}{\left\|\mathbf{b}^{\prime}\right\|} .
\end{aligned}
$$

Therefore, (27) can be changed to the following equation:

$$
1 \otimes \mathbf{a} \oplus 1 \otimes \mathbf{b}=r_{1} \otimes \mathbf{a} \oplus r_{2} \otimes \mathbf{b},
$$

where

$$
\begin{aligned}
& r_{1}=\frac{\tanh ^{-1}\left(t_{1}\left\|\mathbf{a}^{\prime}\right\| / t_{1}^{\prime}\right)}{\tanh ^{-1}\|\mathbf{a}\|}, \\
& r_{2}=\frac{\tanh ^{-1}\left(t_{2}\left\|\mathbf{b}^{\prime}\right\| / t_{2}^{\prime}\right)}{\tanh ^{-1}\|\mathbf{b}\|} .
\end{aligned}
$$

By the previous lemma, we can conclude that $1=r_{1}=r_{2}$, which implies that $\mathbf{a}=\mathbf{a}^{\prime}, \mathbf{b}=\mathbf{b}^{\prime}$. Then, the assumption of our induction shows that $r_{j}=\lambda_{j}(j=1, \ldots, n+1)$. This completes the proof.

Theorem 11. For any finite subset in $\mathbb{V}_{s}$, two notions of linearly independent and gyrolinearly independent coincide.

Proof. $(\Rightarrow)$ It immediately follows from the previous theorem.

$(\Leftarrow)$ We may assume that $s=1$. Assume that the theorem is valid up to $n$, the number of elements of the finite set. Suppose that $\left\{\mathbf{a}_{1}, \ldots, \mathbf{a}_{n+1}\right\} \subset \mathbb{V}_{1}$ is gyrolinearly independent and

$$
t_{1} \frac{\mathbf{a}_{1}}{\left\|\mathbf{a}_{1}\right\|}+\cdots+t_{n+1} \frac{\mathbf{a}_{n+1}}{\left\|\mathbf{a}_{n+1}\right\|}=\mathbf{0} .
$$

By Lemma 8(ii) and the assumption of our induction, it suffices to show that $t_{n+1}=0$. On the contrary, assume that $t_{n+1} \neq 0$. Then, it is obvious that

$$
t_{1} \frac{\mathbf{a}_{1}}{\left\|\mathbf{a}_{1}\right\|}+\cdots+t_{n} \frac{\mathbf{a}_{n}}{\left\|\mathbf{a}_{n}\right\|} \neq \mathbf{0}
$$

Take a positive number $M$ satisfying that

$$
\begin{array}{r}
\frac{t_{1}}{M} \frac{\mathbf{a}_{1}}{\left\|\mathbf{a}_{1}\right\|}+\cdots+\frac{t_{n}}{M} \frac{\mathbf{a}_{n}}{\left\|\mathbf{a}_{n}\right\|} \in \mathbb{V}_{1}, \\
\frac{t_{n+1}}{M} \frac{\mathbf{a}_{n+1}}{\left\|\mathbf{a}_{n+1}\right\|} \in \mathbb{V}_{1} .
\end{array}
$$

Thus we have

$$
\|\mathbf{a}\| \frac{\mathbf{a}}{\|\mathbf{a}\|}+\frac{t_{n+1}}{M} \frac{\mathbf{a}_{n+1}}{\left\|\mathbf{a}_{n+1}\right\|}=\mathbf{0},
$$

where we put

$$
\mathbf{a}=\frac{t_{1}}{M} \frac{\mathbf{a}_{1}}{\left\|\mathbf{a}_{1}\right\|}+\cdots+\frac{t_{n}}{M} \frac{\mathbf{a}_{n}}{\left\|\mathbf{a}_{n}\right\|} .
$$

From [8, Theorem 2.1], we can rewrite (35) in the form of

$$
r \otimes \mathbf{a} \oplus r_{n+1} \otimes \mathbf{a}_{n+1}=\mathbf{0} .
$$

We can also rewrite $r \otimes \mathbf{a}$ in the form of

$$
r \otimes \mathbf{a}=r_{1} \otimes \mathbf{a}_{1} \oplus \cdots \oplus r_{n} \otimes \mathbf{a}_{n}
$$


by using [8, Theorem 3.3], so we obtain the following equation:

$$
\left(r_{1} \otimes \mathbf{a}_{1} \oplus \cdots \oplus r_{n} \otimes \mathbf{a}_{n}\right) \oplus r_{n+1} \otimes \mathbf{a}_{n+1}=\mathbf{0}
$$

Since $\left\{\mathbf{a}_{1}, \ldots, \mathbf{a}_{n+1}\right\}$ is assumed to be gyrolinearly independent, we can conclude that $r_{1}=\cdots=r_{n+1}=0$, which implies that $t_{n+1}=0$. This is a contradiction and completes the proof.

Although the contents in the rest of this section are actually known and used repeatedly in [4], we give their proofs for the convenience of readers.

Lemma 12 (see also [10, Proposition 2.3]).

$$
\|\mathbf{a} \oplus \mathbf{b}\|^{2}=\frac{\|\mathbf{a}\|^{2}+2 \mathbf{a} \cdot \mathbf{b}+\|\mathbf{b}\|^{2}}{1+\left(2 / s^{2}\right) \mathbf{a} \cdot \mathbf{b}+\left(1 / s^{4}\right)\|\mathbf{a}\|^{2}\|\mathbf{b}\|^{2}}
$$

for any $\mathbf{a}, \mathbf{b} \in \mathbb{V}_{s}$.

Proof. By using the definition of $\oplus$, one can easily calculate the inner product of $\mathbf{a} \oplus \mathbf{b}$ with itself to obtain

$$
\begin{aligned}
\|\mathbf{a} \oplus \mathbf{b}\|^{2}=\left(\frac{1}{1+\left(2 / s^{2}\right) \mathbf{a} \cdot \mathbf{b}+\left(1 / s^{4}\right)\|\mathbf{a}\|^{2}\|\mathbf{b}\|^{2}}\right)^{2} \\
\quad \cdot\left\{\left(1+\frac{2}{s^{2}} \mathbf{a} \cdot \mathbf{b}+\frac{1}{s^{2}}\|\mathbf{b}\|^{2}\right)^{2}\|\mathbf{a}\|^{2}\right. \\
+2\left(1+\frac{2}{s^{2}} \mathbf{a} \cdot \mathbf{b}+\frac{1}{s^{2}}\|\mathbf{b}\|^{2}\right)\left(1-\frac{1}{s^{2}}\|\mathbf{a}\|^{2}\right) \mathbf{a} \cdot \mathbf{b} \\
\left.+\left(1-\frac{1}{s^{2}}\|\mathbf{a}\|^{2}\right)^{2}\|\mathbf{b}\|^{2}\right\} .
\end{aligned}
$$

If we put $\mathbf{u}=\mathbf{a} / s, \mathbf{v}=\mathbf{b} / s$, then it is easy to factorize the second factor as

$$
\begin{aligned}
(1+ & \left.+2 \mathbf{u} \cdot \mathbf{v}+\|\mathbf{v}\|^{2}\right)^{2}\|s \mathbf{u}\|^{2}+2\left(1+2 \mathbf{u} \cdot \mathbf{v}+\|\mathbf{v}\|^{2}\right) \\
& \cdot\left(1-\|\mathbf{u}\|^{2}\right) s^{2} \mathbf{u} \cdot \mathbf{v}+\left(1-\|\mathbf{u}\|^{2}\right)^{2}\|s \mathbf{v}\|^{2} \\
& =s^{2}\left(\|\mathbf{u}\|^{2}+2 \mathbf{u} \cdot \mathbf{v}+\|\mathbf{v}\|^{2}\right)\left(1+2 \mathbf{u} \cdot \mathbf{v}+\|\mathbf{u}\|^{2}\|\mathbf{v}\|^{2}\right) \\
& =\left(\|\mathbf{a}\|^{2}+2 \mathbf{a} \cdot \mathbf{b}+\|\mathbf{b}\|^{2}\right) \\
& \cdot\left(1+\frac{2}{s^{2}} \mathbf{a} \cdot \mathbf{b}+\frac{1}{s^{4}}\|\mathbf{a}\|^{2}\|\mathbf{b}\|^{2}\right)
\end{aligned}
$$

hence we can conclude identity (40).

Definition 13 (see [4, Definition 2.7, (2.1)]). Recall that the inverse element of $\mathbf{a}$ is denoted by $\ominus \mathbf{a}$ in a gyrogroup, and one uses the notation

$$
\mathbf{a} \ominus \mathbf{b}=\mathbf{a} \oplus(\ominus \mathbf{b})
$$

as in group theory.
Lemma 14. The following formulae hold:
(i) $(\mathbf{a} / s) \oplus(\mathbf{b} / s)=(\mathbf{a} \oplus \mathbf{b}) / s$.
(ii) $0<1-\left(2 / s^{2}\right) \mathbf{a} \cdot \mathbf{b}+\left(1 / s^{4}\right)\|\mathbf{a}\|^{2}\|\mathbf{b}\|^{2}<2^{2}$.
(iii) $\|\mathbf{a}-\mathbf{b}\| \leq 2\|\mathbf{a} \ominus \mathbf{b}\|$,

for any $\mathbf{a}, \mathbf{b} \in \mathbb{V}_{s}$, where $\oplus$ in the left-hand side of $(i)$ is in the space $\mathbb{V}_{1}$.

Proof. (i) It immediately follows from the definition of $\oplus$.

(ii) By the Cauchy-Schwarz inequality, we have

$$
\begin{aligned}
0 & <\left(1-\frac{1}{s^{2}}\|\mathbf{a}\|\|\mathbf{b}\|\right)^{2} \leq 1-\frac{2}{s^{2}} \mathbf{a} \cdot \mathbf{b}+\frac{1}{s^{4}}\|\mathbf{a}\|^{2}\|\mathbf{b}\|^{2} \\
& \leq 1+\frac{2}{s^{2}}\|\mathbf{a}\|\|\mathbf{b}\|+\frac{1}{s^{4}}\|\mathbf{a}\|^{2}\|\mathbf{b}\|^{2} \\
& =\left(1+\frac{1}{s^{2}}\|\mathbf{a}\|\|\mathbf{b}\|\right)^{2}<2^{2} .
\end{aligned}
$$

(iii) From (ii) just established, identity (40) in Lemma 12, and the fact that $\ominus \mathbf{a}=-\mathbf{a}$ in $\mathbb{V}_{s}$, we have

$$
\begin{aligned}
\frac{\|\mathbf{a}-\mathbf{b}\|^{2}}{2^{2}} & \leq \frac{\|\mathbf{a}\|^{2}-2 \mathbf{a} \cdot \mathbf{b}+\|\mathbf{b}\|^{2}}{1-\left(2 / s^{2}\right) \mathbf{a} \cdot \mathbf{b}+\left(1 / s^{4}\right)\|\mathbf{a}\|^{2}\|\mathbf{b}\|^{2}} \\
& =\|\mathbf{a} \ominus \mathbf{b}\|^{2} .
\end{aligned}
$$

This completes the proof.

Lemma 15 (see [4, Theorem 8.33]). Let $\left\{\mathbf{c}_{j}\right\}_{j=1}^{n}$ be an orthogonal set in $\mathbb{V}_{s}$. Then, for any permutation $\left(i_{1}, \ldots, i_{n}\right)$ of the numbers $\{1, \ldots, n\}$ and any order of gyroaddition for $\mathbf{c}_{i_{1}} \oplus \cdots \oplus$ $\mathbf{c}_{i_{n}}$, the following equality holds:

$$
\frac{\left\|\mathbf{c}_{i_{1}} \oplus \cdots \oplus \mathbf{c}_{i_{n}}\right\|^{2}}{s}=\frac{\left\|\mathbf{c}_{i_{1}}\right\|^{2}}{s} \oplus \cdots \oplus \frac{\left\|\mathbf{c}_{i_{n}}\right\|^{2}}{s} .
$$

Here, $\oplus$ in the right-hand side are in the space $\left\|\mathbb{V}_{s}\right\|$.

Proof. The previous lemma (i) shows that

$$
\left\|\frac{\mathbf{a}}{s} \oplus \frac{\mathbf{b}}{s}\right\|^{2}=\left\|\frac{\mathbf{a} \oplus \mathbf{b}}{s}\right\|^{2}=\frac{\|\mathbf{a} \oplus \mathbf{b}\|^{2}}{s^{2}} .
$$

for any $\mathbf{a}, \mathbf{b}$ in $\mathbb{V}_{s}$. On the other hand, if $\{\mathbf{a}, \mathbf{b}\}$ is orthogonal, then it follows from identity (40) in Lemma 12 that

$$
\begin{aligned}
\left\|\frac{\mathbf{a}}{s} \oplus \frac{\mathbf{b}}{s}\right\|^{2} & =\frac{\|\mathbf{a} / s\|^{2}+\|\mathbf{b} / s\|^{2}}{1+\|\mathbf{a} / s\|^{2}\|\mathbf{b} / s\|^{2}} \\
& =\frac{1}{s} \cdot \frac{\|\mathbf{a}\|^{2} / s+\|\mathbf{b}\|^{2} / s}{1+\left(1 / s^{2}\right)\left(\|\mathbf{a}\|^{2} / s\right)\left(\|\mathbf{b}\|^{2} / s\right)} \\
& =\frac{1}{s}\left(\frac{\|\mathbf{a}\|^{2}}{s} \oplus \frac{\|\mathbf{b}\|^{2}}{s}\right) .
\end{aligned}
$$

Thus the theorem holds for $n=2$. 
Assume that the theorem is valid up to $n$. Let $\left\{\mathbf{c}_{j}\right\}_{j=1}^{n+1}$ be an orthogonal set in $\mathbb{V}_{s}$ and let the following equation

$$
\begin{aligned}
\mathbf{c}_{i_{1}} \oplus \cdots \oplus \mathbf{c}_{i_{n+1}}= & \left(\mathbf{c}_{i_{1}} \oplus \cdots \oplus \mathbf{c}_{i_{m}}\right) \\
& \oplus\left(\mathbf{c}_{i_{m+1}} \oplus \cdots \oplus \mathbf{c}_{i_{n+1}}\right)
\end{aligned}
$$

show the latest gyroaddition $\oplus$. If we put

$$
\begin{aligned}
& \mathbf{a}=\mathbf{c}_{i_{1}} \oplus \cdots \oplus \mathbf{c}_{i_{m}}, \\
& \mathbf{b}=\mathbf{c}_{i_{m+1}} \oplus \cdots \oplus \mathbf{c}_{i_{n+1}},
\end{aligned}
$$

then $\{\mathbf{a}, \mathbf{b}\}$ is orthogonal. From the case of $n=2$, it follows that

$$
\begin{aligned}
\frac{\left\|\mathbf{c}_{i_{1}} \oplus \cdots \oplus \mathbf{c}_{i_{n+1}}\right\|^{2}}{s}= & \frac{\|\mathbf{a} \oplus \mathbf{b}\|^{2}}{s}=\frac{\|\mathbf{a}\|^{2}}{s} \oplus \frac{\|\mathbf{b}\|^{2}}{s} \\
= & \frac{\left\|\mathbf{c}_{i_{1}} \oplus \cdots \oplus \mathbf{c}_{i_{m}}\right\|^{2}}{s} \\
& \oplus \frac{\left\|\mathbf{c}_{i_{m+1}} \oplus \cdots \oplus \mathbf{c}_{i_{n+1}}\right\|^{2}}{s} .
\end{aligned}
$$

Due to the assumption of our induction, we can conclude that

$$
\begin{aligned}
= & \left(\frac{\left\|\mathbf{c}_{i_{1}}\right\|^{2}}{s} \oplus \cdots \oplus \frac{\left\|\mathbf{c}_{i_{m}}\right\|^{2}}{s}\right) \\
& \oplus\left(\frac{\left\|\mathbf{c}_{i_{m+1}}\right\|^{2}}{s} \oplus \cdots \oplus \frac{\left\|\mathbf{c}_{i_{n+1}}\right\|^{2}}{s}\right) .
\end{aligned}
$$

This completes the proof.

\section{The Poincaré Metric and Orthogonal Gyroexpansion in the Möbius Gyrovector Space}

In this section, we give a notion of orthogonal gyroexpansions with respect to a complete orthogonal sequence in the Möbius gyrovector space, which is fully analogous to the notion of the orthogonal expansions with respect to a complete orthonormal sequence in a Hilbert space. It is an application of the orthogonal gyrodecomposition which was established in [8, Theorem 4.2], and we present an explicit procedure to obtain the orthogonal gyroexpansions in the Möbius gyrovector space.

Definition 16 (see [4, Definition 6.8, 6.17 (6.286) and (6.293)]). The Möbius gyrodistance function $d$ on a Möbius gyrovector space $\left(\mathbb{V}_{s}, \oplus, \otimes\right)$ is defined by the equation

$$
d(\mathbf{a}, \mathbf{b})=\|\mathbf{b} \ominus \mathbf{a}\| .
$$

Moreover, the Poincaré distance function $h$ on the ball $\mathbb{V}_{s}$ is introduced by the equation

$$
h(\mathbf{a}, \mathbf{b})=\tanh ^{-1} \frac{d(\mathbf{a}, \mathbf{b})}{s}
$$

for any $\mathbf{a}, \mathbf{b} \in \mathbb{V}_{s}$. Then $h$ satisfies the triangle inequality [4, (6.294)], so that $\left(\mathbb{V}_{s}, h\right)$ is a metric space.

Remark 17. As pointed out in [4, 6.17, p.217], the distance function $h(\mathbf{a}, \mathbf{b})$ is the obvious generalization into the ball $\mathbb{V}_{s}$ of the well-known Poincaré distance function on the disc $\mathbb{D}$. There are a number of literatures dealing with relationship between hyperbolic geometry and Hilbert spaces. In particular, Goebel and Reich [11] introduced the hyperbolic metric $\rho$ on the open unit ball of a complex Hilbert space, from a viewpoint of holomorphic function theory. They developed the study of the Hilbert ball, which leads to research on $\rho$ convexity, nonexpansive mappings, fixed point theorems, and so forth, and [11] is cited in many bibliography such as [12]. The definition of $\rho$ is equivalent to

$$
\rho(x, y)=\tanh ^{-1}(1-\sigma(x, y))^{1 / 2}
$$

where

$$
\sigma(x, y)=\frac{\left(1-\|x\|^{2}\right)\left(1-\|y\|^{2}\right)}{|1-\langle x, y\rangle|^{2}}
$$

for any elements $x, y$ in the Hilbert ball. If we identify $\mathbb{R}^{2}$ with $\mathbb{C}$, then it is easy to see that $h$ and $\rho$ coincide with the Poincaré metric on $\mathbb{D}$. In general, however, $h$ and $\rho$ do not coincide for higher dimensional spaces. We clarify the relationship between $h$ and $\rho$ below.

Lemma 18. Let $\mathbb{V}$ be a real inner product space. Then the norm of the Einstein addition of two elements is given by the equation

$$
\begin{aligned}
& \left\|\mathbf{a} \oplus_{\mathrm{E}} \mathbf{b}\right\|^{2}=\frac{1}{\left(1+(\mathbf{a} \cdot \mathbf{b}) / s^{2}\right)^{2}}\left\{\|\mathbf{a}\|^{2}+\|\mathbf{b}\|^{2}+2 \mathbf{a} \cdot \mathbf{b}\right. \\
& \left.-\frac{1}{s^{2}}\|\mathbf{a}\|^{2}\|\mathbf{b}\|^{2}+\frac{1}{s^{2}}(\mathbf{a} \cdot \mathbf{b})^{2}\right\}
\end{aligned}
$$

for any $\mathbf{a}, \mathbf{b} \in \mathbb{V}_{s}$.

Proof. At first, consider the case $s=1$. From the definition of $\oplus_{\mathrm{E}}$, it is easy to calculate the inner product of $\mathbf{a} \oplus_{\mathrm{E}} \mathbf{b}$ with itself as follows:

$$
\begin{aligned}
& (1+\mathbf{a} \cdot \mathbf{b})^{2}\left\|\mathbf{a} \oplus_{\mathrm{E}} \mathbf{b}\right\|^{2}=\left\langle\mathbf{a}+\frac{1}{\gamma_{\mathbf{a}}} \mathbf{b}+\frac{\gamma_{\mathbf{a}}}{1+\gamma_{\mathbf{a}}}(\mathbf{a} \cdot \mathbf{b}) \mathbf{a}, \mathbf{a}\right. \\
& \left.+\frac{1}{\gamma_{\mathbf{a}}} \mathbf{b}+\frac{\gamma_{\mathbf{a}}}{1+\gamma_{\mathbf{a}}}(\mathbf{a} \cdot \mathbf{b}) \mathbf{a}\right\rangle=\|\mathbf{a}\|^{2}+\frac{1}{\gamma_{\mathbf{a}}^{2}}\|\mathbf{b}\|^{2} \\
& +\left(\frac{\gamma_{\mathbf{a}}}{1+\gamma_{\mathbf{a}}}\right)^{2}(\mathbf{a} \cdot \mathbf{b})^{2}\|\mathbf{a}\|^{2}+\frac{2}{\gamma_{\mathbf{a}}}(\mathbf{a} \cdot \mathbf{b})+\frac{2 \gamma_{\mathbf{a}}}{1+\gamma_{\mathbf{a}}}(\mathbf{a} \\
& \cdot \mathbf{b})\|\mathbf{a}\|^{2}+\frac{2}{1+\gamma_{\mathbf{a}}}(\mathbf{a} \cdot \mathbf{b})^{2}=\|\mathbf{a}\|^{2}+\left(1-\|\mathbf{a}\|^{2}\right)\|\mathbf{b}\|^{2} \\
& +\left(\frac{1 / \sqrt{1-\|\mathbf{a}\|^{2}}}{1+1 / \sqrt{1-\|\mathbf{a}\|^{2}}}\right)^{2}(\mathbf{a} \cdot \mathbf{b})^{2}\|\mathbf{a}\|^{2}
\end{aligned}
$$




$$
\begin{aligned}
& +2 \sqrt{1-\|\mathbf{a}\|^{2}}(\mathbf{a} \cdot \mathbf{b})+\frac{2 \cdot\left(1 / \sqrt{1-\|\mathbf{a}\|^{2}}\right)}{1+1 / \sqrt{1-\|\mathbf{a}\|^{2}}}(\mathbf{a} \cdot \mathbf{b}) \\
& \cdot\|\mathbf{a}\|^{2}+\frac{2}{1+1 / \sqrt{1-\|\mathbf{a}\|^{2}}}(\mathbf{a} \cdot \mathbf{b})^{2}=\|\mathbf{a}\|^{2}+(1 \\
& \left.-\|\mathbf{a}\|^{2}\right)\|\mathbf{b}\|^{2}+\left(\frac{1}{\sqrt{1-\|\mathbf{a}\|^{2}}+1}\right)^{2}(\mathbf{a} \cdot \mathbf{b})^{2}\|\mathbf{a}\|^{2} \\
& +2 \sqrt{1-\|\mathbf{a}\|^{2}}(\mathbf{a} \cdot \mathbf{b})+\frac{2}{\sqrt{1-\|\mathbf{a}\|^{2}}+1}(\mathbf{a} \cdot \mathbf{b})\|\mathbf{a}\|^{2} \\
& +\frac{2 \sqrt{1-\|\mathbf{a}\|^{2}}}{\sqrt{1-\|\mathbf{a}\|^{2}}+1}(\mathbf{a} \cdot \mathbf{b})^{2}=\|\mathbf{a}\|^{2}+\left(1-\|\mathbf{a}\|^{2}\right)\|\mathbf{b}\|^{2} \\
& +\left(\frac{1-\sqrt{1-\|\mathbf{a}\|^{2}}}{\|\mathbf{a}\|^{2}}\right)^{2}(\mathbf{a} \cdot \mathbf{b})^{2}\|\mathbf{a}\|^{2} \\
& +2 \sqrt{1-\|\mathbf{a}\|^{2}}(\mathbf{a} \cdot \mathbf{b})+\frac{2\left(1-\sqrt{1-\|\mathbf{a}\|^{2}}\right)}{\|\mathbf{a}\|^{2}}(\mathbf{a} \cdot \mathbf{b}) \\
& \cdot\|\mathbf{a}\|^{2}+\frac{2 \sqrt{1-\|\mathbf{a}\|^{2}}\left(1-\sqrt{1-\|\mathbf{a}\|^{2}}\right)}{\|\mathbf{a}\|^{2}}(\mathbf{a} \cdot \mathbf{b})^{2} \\
& =\|\mathbf{a}\|^{2}+\|\mathbf{b}\|^{2}+2 \mathbf{a} \cdot \mathbf{b}-\|\mathbf{a}\|^{2}\|\mathbf{b}\|^{2}+(\mathbf{a} \cdot \mathbf{b})^{2} .
\end{aligned}
$$

Thus the lemma holds for $s=1$. For general $s>0$, let $\mathbf{a}, \mathbf{b} \in$ $\mathbb{V}_{s}$. If we put $\mathbf{u}=\mathbf{a} / s$ and $\mathbf{v}=\mathbf{b} / s$, then it is immediate to see that

$$
\mathbf{u} \oplus_{\mathrm{E}} \mathbf{v}=\frac{\mathbf{a} \oplus_{\mathrm{E}} \mathbf{b}}{s},
$$

and we can easily deduce identity (57) by applying the case $s=1$ to $\mathbf{u}, \mathbf{v}$. This completes the proof.

Theorem 19. Let one use the notations $\rho$ and $\sigma$ in $\mathbb{V}_{s}$ by

$$
\rho(\mathbf{a}, \mathbf{b})=\tanh ^{-1}(1-\sigma(\mathbf{a}, \mathbf{b}))^{1 / 2},
$$

where

$$
\sigma(\mathbf{a}, \mathbf{b})=\frac{\left(s^{2}-\|\mathbf{a}\|^{2}\right)\left(s^{2}-\|\mathbf{b}\|^{2}\right)}{\left(s^{2}-\mathbf{a} \cdot \mathbf{b}\right)^{2}}
$$

for $\mathbf{a}, \mathbf{b} \in \mathbb{V}_{s}$. Then the following identities hold:

(i) $\left\|\mathbf{a} \ominus_{\mathrm{E}} \mathbf{b}\right\| / s=(1-\sigma(\mathbf{a}, \mathbf{b}))^{1 / 2}$.

(ii) $h_{\mathrm{E}}(\mathbf{a}, \mathbf{b})=\tanh ^{-1}\left(\left\|\mathbf{a} \ominus_{\mathrm{E}} \mathbf{b}\right\| / s\right)=\rho(\mathbf{a}, \mathbf{b})$.

(iii) $2 h(\mathbf{a}, \mathbf{b})=2 \tanh ^{-1}(\|\mathbf{a} \ominus \mathbf{b}\| / s)=\rho(2 \otimes \mathbf{a}, 2 \otimes \mathbf{b})$.

Proof. (i) and (ii) immediately follow from the previous lemma. (iii) It is not difficult to see that we may assume $s=1$. By (ii) just established, it suffices to show that

$$
2 \otimes\|\mathbf{a} \ominus \mathbf{b}\|=\left\|2 \otimes \mathbf{a} \ominus_{\mathrm{E}} 2 \otimes \mathbf{b}\right\|
$$

Note that

$$
2 \otimes a=\frac{(1+a)^{2}-(1-a)^{2}}{(1+a)^{2}+(1-a)^{2}}=\frac{2 a}{1+a^{2}}
$$

for real number $0 \leq a<1$. For any $\mathbf{a}, \mathbf{b} \in \mathbb{V}_{1}$, if we put $a=\|\mathbf{a}\|$, $b=\|\mathbf{b}\|$, and $\alpha=(\mathbf{a} /\|\mathbf{a}\|) \cdot(\mathbf{b} /\|\mathbf{b}\|)$, then, by the definition or the axioms of gyrovector spaces, we have

$$
\begin{aligned}
\|2 \otimes \mathbf{a}\| & =2 \otimes\|\mathbf{a}\|=\frac{2 a}{1+a^{2}}, \\
(2 \otimes \mathbf{a}) \cdot(2 \otimes \mathbf{b}) & =\frac{2 a}{1+a^{2}} \cdot \frac{2 b}{1+b^{2}} \cdot \alpha .
\end{aligned}
$$

By identity (40) in Lemma 12,

$$
\begin{aligned}
2 \otimes & \|\mathbf{a} \ominus \mathbf{b}\|=2 \otimes \sqrt{\frac{\|\mathbf{a}\|^{2}-2 \mathbf{a} \cdot \mathbf{b}+\|\mathbf{b}\|^{2}}{1-2 \mathbf{a} \cdot \mathbf{b}+\|\mathbf{a}\|^{2}\|\mathbf{b}\|^{2}}} \\
& =\frac{2 \sqrt{\left(a^{2}-2 a b \alpha+b^{2}\right) /\left(1-2 a b \alpha+a^{2} b^{2}\right)}}{1+\left(a^{2}-2 a b \alpha+b^{2}\right) /\left(1-2 a b \alpha+a^{2} b^{2}\right)} \\
& =\frac{2 \sqrt{\left(a^{2}-2 a b \alpha+b^{2}\right)\left(1-2 a b \alpha+a^{2} b^{2}\right)}}{\left(1-2 a b \alpha+a^{2} b^{2}\right)+\left(a^{2}-2 a b \alpha+b^{2}\right)} .
\end{aligned}
$$

On the other hand, identity (57) in the previous lemma shows that

$$
\begin{aligned}
& \left\|2 \otimes \mathbf{a} \ominus_{\mathrm{E}} 2 \otimes \mathbf{b}\right\|=\sqrt{\frac{\|2 \otimes \mathbf{a}\|^{2}+\|2 \otimes \mathbf{b}\|^{2}-2(2 \otimes \mathbf{a}) \cdot(2 \otimes \mathbf{b})-\|2 \otimes \mathbf{a}\|^{2}\|2 \otimes \mathbf{b}\|^{2}+\{(2 \otimes \mathbf{a}) \cdot(2 \otimes \mathbf{b})\}^{2}}{\{1-(2 \otimes \mathbf{a}) \cdot(2 \otimes \mathbf{b})\}^{2}}} \\
& =\frac{\sqrt{\left(2 a /\left(1+a^{2}\right)\right)^{2}+\left(2 b /\left(1+b^{2}\right)\right)^{2}-2 \cdot\left(2 a /\left(1+a^{2}\right)\right) \cdot\left(2 b /\left(1+b^{2}\right)\right) \cdot \alpha-\left(2 a /\left(1+a^{2}\right)\right)^{2}\left(2 b /\left(1+b^{2}\right)\right)^{2}+\left\{\left(2 a /\left(1+a^{2}\right)\right) \cdot\left(2 b /\left(1+b^{2}\right)\right) \cdot \alpha\right\}^{2}}}{1-\left(2 a /\left(1+a^{2}\right)\right) \cdot\left(2 b /\left(1+b^{2}\right)\right) \cdot \alpha} \\
& =\frac{2 \sqrt{\left(a^{2}-2 a b \alpha+b^{2}\right)\left(1-2 a b \alpha+a^{2} b^{2}\right)}}{\left(1+a^{2}\right)\left(1+b^{2}\right)-4 a b \alpha} .
\end{aligned}
$$

This completes the proof. 
In the rest of the paper, we should concentrate to investigate the Möbius ball endowed with the Poincaré metric $h$ introduced by Ungar. We can perform gyrolinear algebraic operations which behave quite well for orthogonal sequences in the Möbius gyrovector spaces, like as linear algebraic ones in Hilbert spaces.

Lemma 20. For any sequence $\left\{\mathbf{a}_{n}\right\}_{n=1}^{\infty}$ and any element $\mathbf{a}$ in $\mathbb{V}_{s}$,

(i) $h\left(\mathbf{a}_{n}, \mathbf{a}\right) \rightarrow 0(n \rightarrow \infty) \Leftrightarrow d\left(\mathbf{a}_{n}, \mathbf{a}\right) \rightarrow 0(n \rightarrow \infty)$,

(ii) $h\left(\mathbf{a}_{n}, \mathbf{a}_{m}\right) \rightarrow 0(n, m \rightarrow \infty) \Leftrightarrow d\left(\mathbf{a}_{n}, \mathbf{a}_{m}\right) \rightarrow$ $0(n, m \rightarrow \infty)$.

Proof. It is obvious, because both $\tanh$ and $\tanh ^{-1}$ are uniformly continuous on a neighborhood of 0 .

Lemma 21. For any fixed $\mathbf{a} \in \mathbb{V}_{s}$, the map $\mathbb{V}_{s} \ni \mathbf{x} \mapsto \mathbf{x} \cdot \mathbf{a} \in$ $\left(-s^{2}, s^{2}\right)$ is continuous, where one considers the metrich on both sets.

Proof. Suppose that $h\left(\mathbf{x}_{n}, \mathbf{x}\right) \rightarrow 0$. Then, from the previous lemma and Lemma 14(iii), it follows that $\left\|\mathbf{x}_{n}-\mathbf{x}\right\| \leq 2 \| \mathbf{x}_{n} \ominus$ $\mathbf{x} \| \rightarrow 0$. Therefore, we have

$$
\begin{aligned}
&\left|\mathbf{x}_{n} \cdot \mathbf{a} \ominus \mathbf{x} \cdot \mathbf{a}\right|=\left|\frac{\mathbf{x}_{n} \cdot \mathbf{a}-\mathbf{x} \cdot \mathbf{a}}{1-\left(1 / s^{4}\right)\left(\mathbf{x}_{n} \cdot \mathbf{a}\right)(\mathbf{x} \cdot \mathbf{a})}\right| \longrightarrow 0 \\
&(n \longrightarrow \infty) .
\end{aligned}
$$

This implies that $h\left(\mathbf{x}_{n} \cdot \mathbf{a}, \mathbf{x} \cdot \mathbf{a}\right) \rightarrow 0$.

We should make sure of two definitions here. One of them is quite usual; another is very natural.

For any nonempty subset $A$ of $\mathbb{V}_{s}$, we denote $A^{\perp}$ as the orthogonal complement of $A$ in $\mathbb{V}$; that is,

$$
A^{\perp}=\{\mathbf{x} \in \mathbb{V} ; \mathbf{x} \cdot \mathbf{a}=0 \forall \mathbf{a} \in A\} .
$$

A nonempty subset $M$ of $\mathbb{V}_{s}$ is a gyrovector subspace if $M$ is closed under gyroaddition and gyroscalar multiplication; that is, $\mathbf{a}, \mathbf{b} \in M$ and $r \in \mathbb{R}$ imply that $\mathbf{a} \oplus \mathbf{b} \in M$ and $r \otimes \mathbf{a} \in M$.

Lemma 22. $A^{\perp} \cap \mathbb{V}_{s}$ is an h-closed gyrovector subspace.

Proof. From the definitions of $\oplus$ and $\otimes$, it is immediate to see that $A^{\perp} \cap \mathbb{V}_{s}$ forms a gyrovector subspace. Moreover, $A^{\perp} \cap \mathbb{V}_{s}$ is obviously $h$-closed by the previous lemma.

Lemma 23. If $\mathbf{a}_{n}, \mathbf{a} \in \mathbb{V}_{s}$ and $\left\|\mathbf{a}_{n}-\mathbf{a}\right\| \rightarrow 0$, then $h\left(\mathbf{a}_{n}, \mathbf{a}\right) \rightarrow 0$.

Proof. It suffices to show that $d\left(\mathbf{a}_{n}, \mathbf{a}\right) \rightarrow 0$. By the assumption $\|\mathbf{a}\|<s$, we can obtain

$$
\begin{aligned}
d\left(\mathbf{a}_{n}, \mathbf{a}\right)^{2} & =\left\|\mathbf{a}_{n} \ominus \mathbf{a}\right\|^{2} \\
& =\frac{\left\|\mathbf{a}_{n}\right\|^{2}-2 \mathbf{a}_{n} \cdot \mathbf{a}+\|\mathbf{a}\|^{2}}{1-\left(2 / s^{2}\right) \mathbf{a}_{n} \cdot \mathbf{a}+\left(1 / s^{4}\right)\left\|\mathbf{a}_{n}\right\|^{2}\|\mathbf{a}\|^{2}} \\
& \longrightarrow 0 \quad(n \longrightarrow \infty),
\end{aligned}
$$

where we used identity (40) in Lemma 12.
Proposition 24. Let $M$ be a gyrovector subspace of $\mathbb{V}_{s}$. Then the closure $\vec{M}^{h}$ with respect to the metric $h$ is also a gyrovector subspace.

Proof. Suppose that $\mathbf{a}, \mathbf{b} \in \bar{M}^{h}$. There exist sequences $\left\{\mathbf{a}_{n}\right\},\left\{\mathbf{b}_{n}\right\} \subset M$ such that $h\left(\mathbf{a}_{n}, \mathbf{a}\right), h\left(\mathbf{b}_{n}, \mathbf{b}\right) \rightarrow 0$. By Lemmas 20 and 14(iii), we have $\left\|\mathbf{a}_{n}-\mathbf{a}\right\|,\left\|\mathbf{b}_{n}-\mathbf{b}\right\| \rightarrow 0$. From the definitions of $\oplus, \otimes$, it is easy to see that $\left\|\left(\mathbf{a}_{n} \oplus \mathbf{b}_{n}\right)-(\mathbf{a} \oplus \mathbf{b})\right\| \rightarrow 0$ and $\left\|r \otimes \mathbf{a}_{n}-r \otimes \mathbf{a}\right\| \rightarrow 0$. By Lemma 23, it follows that $h\left(\mathbf{a}_{n} \oplus \mathbf{b}_{n}, \mathbf{a} \oplus \mathbf{b}\right) \rightarrow 0$ and $h\left(r \otimes \mathbf{a}_{n}, r \otimes \mathbf{a}\right) \rightarrow 0$. Since $\mathbf{a}_{n} \oplus \mathbf{b}_{n}, r \otimes \mathbf{a}_{n} \in M$, we can conclude that $\mathbf{a} \oplus \mathbf{b}, r \otimes \mathbf{a} \in \bar{M}^{h}$. This completes the proof.

Lemma 25. Any finitely generated gyrovector subspace is $h$ closed.

Proof. Let $M$ be a gyrovector subspace generated by nonzero elements $\left\{\mathbf{a}_{1}, \ldots, \mathbf{a}_{n}\right\}$ in $\mathbb{V}_{s}$. For an arbitrary element $\mathbf{x} \in \bar{M}^{h}$, there exists a sequence $\left\{\mathbf{x}_{k}\right\}_{k=1}^{\infty} \subset M$ such that $h\left(\mathbf{x}_{k}, \mathbf{x}\right) \rightarrow 0$. Then, from Lemmas 20(i) and 14(iii), it follows that $\left\|\mathbf{x}_{k}-\mathbf{x}\right\| \rightarrow$ 0 . By $[8$, Theorem 3.3], we have

$$
M=\left\{t_{1} \frac{\mathbf{a}_{1}}{\left\|\mathbf{a}_{1}\right\|}+\cdots+t_{n} \frac{\mathbf{a}_{n}}{\left\|\mathbf{a}_{n}\right\|} ; t_{1}, \ldots, t_{n} \in \mathbb{R}\right\} \cap \mathbb{V}_{s} .
$$

Since $\left\{t_{1}\left(\mathbf{a}_{1} /\left\|\mathbf{a}_{1}\right\|\right)+\cdots+t_{n}\left(\mathbf{a}_{n} /\left\|\mathbf{a}_{n}\right\|\right) ; t_{1}, \ldots, t_{n} \in \mathbb{R}\right\}$ is a finite dimensional linear subspace, it is closed with respect to the norm topology. Therefore $\mathbf{x} \in M$. This completes the proof.

From now on, we assume that the carrier $\mathbb{V}$ of the Möbius gyrovector space $\mathbb{V}_{s}$ is complete as a metric space with respect to the norm induced by the inner product. Thus, $\mathbb{V}$ is a real Hilbert space.

Theorem 26. Let $\mathbb{V}$ be a real Hilbert space. Then $\left(\mathbb{V}_{s}, h\right)$ is a complete metric space.

Although this fact is well-known and it can be deduced by existing results and Theorem 19, we give a direct proof here in order to show how gyrovector space approach is fully analogous to vector space approach.

Proof. Without loss of generality, we may assume that $s=1$. Suppose that $\left\{\mathbf{a}_{n}\right\}_{n=1}^{\infty}$ is a Cauchy sequence in $\left(\mathbb{V}_{1}, h\right)$. From Lemmas 14(iii) and 20(ii), it follows that

$$
\begin{aligned}
\left\|\mathbf{a}_{n}-\mathbf{a}_{m}\right\| \leq 2\left\|\mathbf{a}_{n} \ominus \mathbf{a}_{m}\right\|=2 d\left(\mathbf{a}_{n}, \mathbf{a}_{m}\right) & \longrightarrow 0 \\
& (n, m \longrightarrow \infty),
\end{aligned}
$$

which implies that $\left\{\mathbf{a}_{n}\right\}$ is a Cauchy sequence with respect to the norm of $\mathbb{V}$. Hence there exists a unique element $\mathbf{a} \in \mathbb{V}$ such that $\|\mathbf{a}\| \leq 1,\left\|\mathbf{a}_{n}-\mathbf{a}\right\| \rightarrow 0$. In order to show that $\|\mathbf{a}\|<1$, on the contrary, we assume that $\|\mathbf{a}\|=1$. By the assumption that $\left\{\mathbf{a}_{n}\right\}$ is a Cauchy sequence in $\left(\mathbb{V}_{1}, h\right)$, there exists a natural number $m_{0}$ such that

$$
d\left(\mathbf{a}_{m+p}, \mathbf{a}_{m}\right)^{2}<\frac{1}{2}
$$


for any $m \geq m_{0}$ and any $p$. On the other hand, from identity (40) in Lemma 12, we have

$$
d\left(\mathbf{a}_{m+p}, \mathbf{a}_{m}\right)^{2}=\frac{\left\|\mathbf{a}_{m+p}\right\|^{2}-2 \mathbf{a}_{m+p} \cdot \mathbf{a}_{m}+\left\|\mathbf{a}_{m}\right\|^{2}}{1-2 \mathbf{a}_{m+p} \cdot \mathbf{a}_{m}+\left\|\mathbf{a}_{m+p}\right\|^{2}\left\|\mathbf{a}_{m}\right\|^{2}}
$$

Now we fix $m \geq m_{0}$ and let $p \rightarrow \infty$. Then, from the fact that $\mathbf{a}_{m+p} \rightarrow \mathbf{a}$ and the assumption $\|\mathbf{a}\|=1$, we can obtain

$$
\frac{1}{2} \geq \frac{\|\mathbf{a}\|^{2}-2 \mathbf{a} \cdot \mathbf{a}_{m}+\left\|\mathbf{a}_{m}\right\|^{2}}{1-2 \mathbf{a} \cdot \mathbf{a}_{m}+\|\mathbf{a}\|^{2}\left\|\mathbf{a}_{m}\right\|^{2}}=1
$$

which is a contradiction. This implies that $\|\mathbf{a}\|<1$. By Lemma 23, the proof is complete.

Theorem 27. Let $\mathbb{V}$ be a real Hilbert space. If $A$ is a closed subset in $\left(\mathbb{V}_{s}, h\right)$, then $A$ is relatively closed in $\left(\mathbb{V}_{s},\|\cdot\|\right)$. Therefore, the orthogonal gyrodecomposition is applicable to $h$ closed gyrovector subspaces in the sense of [8, Theorem 4.2].

Proof. Denote by $\bar{A}$ the closure of $A$ with respect to the norm topology. It suffices to show that $A=\bar{A} \cap \mathbb{V}_{s}$. One of the inclusions $(c)$ is trivial. If $\mathbf{x} \in \bar{A} \cap \mathbb{V}_{s}$, then there exists a sequence $\left\{\mathbf{x}_{n}\right\} \subset A$ such that $\left\|\mathbf{x}_{n}-\mathbf{x}\right\| \rightarrow 0$. So we can apply Lemma 23 to obtain that $h\left(\mathbf{x}_{n}, \mathbf{x}\right) \rightarrow 0$. Hence $\mathbf{x} \in A$ and this completes the proof.

Theorem 28. Let $M$ be a gyrovector subspace of $\mathbb{V}_{s}$ and $\mathbf{x} \in \mathbb{V}_{s}$. Then one has

$$
\mathbf{x} \in M^{\perp} \Longleftrightarrow\|\mathbf{x} \ominus \mathbf{m}\| \geq\|\mathbf{x}\| \quad(\mathbf{m} \in M) .
$$

Proof. $(\Rightarrow)$ Suppose that $\mathbf{x} \in M^{\perp} \cap \mathbb{V}_{s}, \mathbf{m} \in M$. By identity (40) in Lemma 12, we obtain

$$
\|\mathbf{x} \ominus \mathbf{m}\|^{2}=\frac{\|\mathbf{x}\|^{2}+\|\mathbf{m}\|^{2}}{1+\left(1 / s^{4}\right)\|\mathbf{x}\|^{2}\|\mathbf{m}\|^{2}} \geq\|\mathbf{x}\|^{2} .
$$

$(\Leftarrow)$ Suppose that $\mathbf{m} \in M$. For an arbitrary positive real number $t$, take

$$
r=t \frac{|\mathbf{x} \cdot \mathbf{m}|}{\mathbf{x} \cdot \mathbf{m}}
$$

Since $r \otimes \mathbf{m} \in M$, it follows from identity (40) in Lemma 12 that

$$
\begin{aligned}
\|\mathbf{x}\|^{2} & \leq\|\mathbf{x} \ominus(r \otimes \mathbf{m})\|^{2} \\
& =\frac{\|\mathbf{x}\|^{2}-2 \mathbf{x} \cdot(r \otimes \mathbf{m})+\|r \otimes \mathbf{m}\|^{2}}{1-\left(2 / s^{2}\right) \mathbf{x} \cdot(r \otimes \mathbf{m})+\left(1 / s^{4}\right)\|\mathbf{x}\|^{2}\|r \otimes \mathbf{m}\|^{2}} .
\end{aligned}
$$

By the axiom (V7), we have $\|r \otimes \mathbf{m}\|=|r| \otimes\|\mathbf{m}\|=t \otimes\|\mathbf{m}\|$ and the inequality

$$
\begin{aligned}
& \|\mathbf{x}\|^{2}\left\{1-\frac{2}{s^{2}} \mathbf{x} \cdot(r \otimes \mathbf{m})+\frac{1}{s^{4}}\|\mathbf{x}\|^{2}(t \otimes\|\mathbf{m}\|)^{2}\right\} \\
& \leq\|\mathbf{x}\|^{2}-2 \mathbf{x} \cdot(r \otimes \mathbf{m})+(t \otimes\|\mathbf{m}\|)^{2},
\end{aligned}
$$

which yields the following inequality:

$$
2 \mathbf{x} \cdot(r \otimes \mathbf{m}) \leq\left(1+\frac{1}{s^{2}}\|\mathbf{x}\|^{2}\right)(t \otimes\|\mathbf{m}\|)^{2} ;
$$

namely,

$$
\begin{aligned}
& 2 s \tanh \left(r \tanh ^{-1} \frac{\|\mathbf{m}\|}{s}\right) \frac{\mathbf{x} \cdot \mathbf{m}}{\|\mathbf{m}\|} \\
& \quad \leq\left(1+\frac{1}{s^{2}}\|\mathbf{x}\|^{2}\right)\left(s \tanh \left(t \tanh ^{-1} \frac{\|\mathbf{m}\|}{s}\right)\right)^{2} .
\end{aligned}
$$

Note that $\mathbf{x} \cdot \mathbf{m}$ and $r$ have the same signature, so we have

$$
\begin{aligned}
& \tanh \left(r \tanh ^{-1} \frac{\|\mathbf{m}\|}{s}\right) \mathbf{x} \cdot \mathbf{m} \\
& \quad=\tanh \left(t \tanh ^{-1} \frac{\|\mathbf{m}\|}{s}\right)|\mathbf{x} \cdot \mathbf{m}| .
\end{aligned}
$$

Therefore, we can obtain the inequality

$$
2 \frac{|\mathbf{x} \cdot \mathbf{m}|}{\|\mathbf{m}\|} \leq\left(1+\frac{1}{s^{2}}\|\mathbf{x}\|^{2}\right) s \tanh \left(t \tanh ^{-1} \frac{\|\mathbf{m}\|}{s}\right) .
$$

Since $t>0$ is arbitrary, we can let $t \rightarrow+0$ and conclude that $\mathbf{x} \cdot \mathbf{m}=0$. This completes the proof.

Lemma 29. In a gyrocommutative gyrogroup, one has

$$
\mathbf{a} \ominus(\mathbf{b} \oplus \mathbf{c})=\operatorname{gyr}[\mathbf{a}, \ominus \mathbf{b}]\{(\ominus \mathbf{b} \oplus \mathbf{a}) \ominus \mathbf{c}\} .
$$

This lemma can be obtained if we put $a, b$ and $c$ as $\ominus \mathbf{b}, \mathbf{a}$ and $\ominus \mathbf{c}$, respectively, in [4, Theorem 3.9]. However, we give a proof for the convenience of readers by using gyroautomorphic inverse property $\ominus(\mathbf{a} \oplus \mathbf{b})=\ominus \mathbf{a} \ominus \mathbf{b}$, left gyroassociative law (G3), gyrocommutativity (G6), and gyroautomorphism (G4).

Proof.

$$
\begin{aligned}
\mathbf{a} \ominus(\mathbf{b} \oplus \mathbf{c}) & =\mathbf{a} \oplus(\ominus \mathbf{b} \ominus \mathbf{c}) \\
& =(\mathbf{a} \oplus(\ominus \mathbf{b})) \oplus \operatorname{gyr}[\mathbf{a}, \ominus \mathbf{b}](\ominus \mathbf{c}) \\
& =\operatorname{gyr}[\mathbf{a}, \ominus \mathbf{b}](\ominus \mathbf{b} \oplus \mathbf{a}) \oplus \operatorname{gyr}[\mathbf{a}, \ominus \mathbf{b}](\ominus \mathbf{c}) \\
& =\operatorname{gyr}[\mathbf{a}, \ominus \mathbf{b}]\{(\ominus \mathbf{b} \oplus \mathbf{a}) \oplus(\ominus \mathbf{c})\} .
\end{aligned}
$$

Theorem 30. Let $M$ be an h-closed gyrovector subspace of $\mathbb{V}_{s}$ and $\mathbf{x} \in \mathbb{V}_{s}$.

(i) Let

$$
\mathbf{x}=\mathbf{y} \oplus \mathbf{z}, \quad \mathbf{y} \in M, \mathbf{z} \in M^{\perp} \cap \mathbb{V}_{s}
$$

be the orthogonal gyrodecomposition of $\mathbf{x} \in \mathbb{V}_{s}$ by Theorem 27 and [8, Theorem 4.2]. Then $\mathbf{y}$ is the closest point to $\mathbf{x}$ in $M$. Thus $\mathbf{y}$ satisfies the identity

$$
h(\mathbf{x}, \mathbf{y})=\inf _{\mathbf{m} \in M} h(\mathbf{x}, \mathbf{m}) .
$$


(ii) Conversely, let $\mathbf{y}$ be the closest point to $\mathbf{x}$ in $M$; namely, $\mathbf{y}$ is an element in $M$ satisfying identity (87). Then

$$
\mathbf{x}=\mathbf{y} \oplus(\ominus \mathbf{y} \oplus \mathbf{x})
$$

is the orthogonal gyrodecomposition. Thus $\ominus \mathbf{y} \oplus \mathbf{x} \in$ $M^{\perp} \cap \mathbb{V}_{s}$.

Proof. Note that $h\left(\mathbf{a}_{1}, \mathbf{a}_{2}\right) \leq h\left(\mathbf{b}_{1}, \mathbf{b}_{2}\right)$ if and only if $\left\|\mathbf{a}_{1} \ominus \mathbf{a}_{2}\right\| \leq$ $\left\|\mathbf{b}_{1} \ominus \mathbf{b}_{2}\right\|$

(i) Suppose that $\mathbf{x}=\mathbf{y} \oplus \mathbf{z}, \mathbf{y} \in M, \mathbf{z} \in M^{\perp} \cap \mathbb{V}_{s}$. For any $\mathbf{m} \in M$, we have

$$
\begin{aligned}
\mathbf{x} \ominus \mathbf{m} & =\mathbf{x} \ominus(\mathbf{y} \oplus(\ominus \mathbf{y} \oplus \mathbf{m})) \\
& =\operatorname{gyr}[\mathbf{x}, \ominus \mathbf{y}]\{(\ominus \mathbf{y} \oplus \mathbf{x}) \ominus(\ominus \mathbf{y} \oplus \mathbf{m})\} \\
& =\operatorname{gyr}[\mathbf{x}, \ominus \mathbf{y}]\{\mathbf{z} \ominus(\ominus \mathbf{y} \oplus \mathbf{m})\}
\end{aligned}
$$

by the previous lemma. From $\ominus \mathbf{y} \oplus \mathbf{x}=\mathbf{z} \in M^{\perp} \cap \mathbb{V}_{s}, \ominus \mathbf{y} \oplus \mathbf{m} \in$ $M$ and Theorem 28, it follows that

$$
\begin{aligned}
\|\mathbf{x} \ominus \mathbf{y}\| & =\|\mathbf{z}\| \leq\|\mathbf{z} \ominus(\ominus \mathbf{y} \oplus \mathbf{m})\| \\
& =\|\operatorname{gyr}[\mathbf{x}, \ominus \mathbf{y}](\mathbf{z} \ominus(\ominus \mathbf{y} \oplus \mathbf{m}))\|=\|\mathbf{x} \ominus \mathbf{m}\|,
\end{aligned}
$$

because each gyroautomorphism preserves the norm. Since $\mathbf{m} \in M$ is arbitrary, we can conclude $\|\mathbf{x} \ominus \mathbf{y}\| \leq \inf _{\mathbf{m} \in M} \| \mathbf{x} \ominus$ $\mathbf{m} \|$, and the opposite inequality trivially holds. Thus y satisfies identity (87).

(ii) Put $\mathbf{z}=\ominus \mathbf{y} \oplus \mathbf{x}$. For any $\mathbf{m} \in M$, we have

$$
\begin{aligned}
\mathbf{x} \ominus(\mathbf{y} \oplus \mathbf{m}) & =\operatorname{gyr}[\mathbf{x}, \ominus \mathbf{y}]\{(\ominus \mathbf{y} \oplus \mathbf{x}) \ominus \mathbf{m}\} \\
& =\operatorname{gyr}[\mathbf{x}, \ominus \mathbf{y}](\mathbf{z} \ominus \mathbf{m})
\end{aligned}
$$

by the previous lemma. From $\mathbf{y} \oplus \mathbf{m} \in M$ and identity (87), it follows that

$$
\begin{aligned}
\|\mathbf{z}\| & =\|\ominus \mathbf{y} \oplus \mathbf{x}\|=\|\mathbf{x} \ominus \mathbf{y}\| \leq\|\mathbf{x} \ominus(\mathbf{y} \oplus \mathbf{m})\| \\
& =\|\operatorname{gyr}[\mathbf{x}, \ominus \mathbf{y}](\mathbf{z} \ominus \mathbf{m})\|=\|\mathbf{z} \ominus \mathbf{m}\|,
\end{aligned}
$$

because each gyroautomorphism preserves the norm. Thus we can apply Theorem 28 and obtain that $\mathbf{z} \in M^{\perp}$. This completes the proof.

The following lemma plays a key role in our orthogonal gyroexpansion.

Lemma 31. If $\{\mathbf{u}, \mathbf{v}, \mathbf{w}\}$ is an orthogonal set in $\mathbb{V}_{s}$, then the associative law holds; that is,

$$
\mathbf{u} \oplus(\mathbf{v} \oplus \mathbf{w})=(\mathbf{u} \oplus \mathbf{v}) \oplus \mathbf{w}
$$

Proof. By [4, (3.147), (3.148)], the gyration in the Möbius gyrovector spaces $\mathbb{V}_{s}$ can be expressed by the equation

$$
\operatorname{gyr}[\mathbf{u}, \mathbf{v}] \mathbf{w}=\mathbf{w}+2 \frac{A \mathbf{u}+B \mathbf{v}}{D}
$$

where

$$
\begin{aligned}
& A=-\frac{1}{s^{4}} \mathbf{u} \cdot \mathbf{w}\|\mathbf{v}\|^{2}+\frac{1}{s^{2}} \mathbf{v} \cdot \mathbf{w}+\frac{2}{s^{4}}(\mathbf{u} \cdot \mathbf{v})(\mathbf{v} \cdot \mathbf{w}) \\
& B=-\frac{1}{s^{4}} \mathbf{v} \cdot \mathbf{w}\|\mathbf{u}\|^{2}-\frac{1}{s^{2}} \mathbf{u} \cdot \mathbf{w} \\
& D=1+\frac{2}{s^{2}} \mathbf{u} \cdot \mathbf{v}+\frac{1}{s^{4}}\|\mathbf{u}\|^{2}\|\mathbf{v}\|^{2}
\end{aligned}
$$

for all $\mathbf{u}, \mathbf{v}, \mathbf{w} \in \mathbb{V}_{s}$. See also [10, Proposition 2.14] for a proof by hand calculation. If $\{\mathbf{u}, \mathbf{v}, \mathbf{w}\}$ is orthogonal, then we have $\mathrm{A}=\mathrm{B}=0$, so that $\operatorname{gyr}[\mathbf{u}, \mathbf{v}] \mathbf{w}=\mathbf{w}$. This completes the proof.

Definition 32. (i) Let $\left\{\mathbf{a}_{n}\right\}_{n}$ be a sequence in $\mathbb{V}_{s}$. One says that a series

$$
\left(\left(\left(\mathbf{a}_{1} \oplus \mathbf{a}_{2}\right) \oplus \mathbf{a}_{3}\right) \oplus \cdots \oplus \mathbf{a}_{n}\right) \oplus \cdots
$$

converges if there exists an element $\mathbf{x} \in \mathbb{V}_{s}$ such that $h\left(\mathbf{x}, \mathbf{x}_{n}\right) \rightarrow 0(n \rightarrow \infty)$, where the sequence $\left\{\mathbf{x}_{n}\right\}_{n}$ is defined recursively by $\mathbf{x}_{1}=\mathbf{a}_{1}$ and $\mathbf{x}_{n}=\mathbf{x}_{n-1} \oplus \mathbf{a}_{n}$. In this case, we say the series converges to $\mathbf{x}$ and denote

$$
\mathbf{x}=\left(\left(\left(\mathbf{a}_{1} \oplus \mathbf{a}_{2}\right) \oplus \mathbf{a}_{3}\right) \oplus \cdots \oplus \mathbf{a}_{n}\right) \oplus \cdots .
$$

(ii) Let $\left\{a_{n}\right\}_{n}$ be a sequence in $\mathbb{R}$ with $\left|a_{n}\right|<s$ for all $n$. We say that a series

$$
\sum_{n=1}^{\infty} a_{n}=a_{1} \oplus a_{2} \oplus \cdots \oplus a_{n} \oplus \cdots
$$

converges if there exists $x \in \mathbb{R}$ with $|x|<s$ such that $x_{n} \rightarrow x$, where the sequence $\left\{x_{n}\right\}_{n}$ is defined recursively by $x_{1}=a_{1}$ and $x_{n}=x_{n-1} \oplus a_{n}$. In this case, we say the series converges to $x$ and denote

$$
x=\sum_{n=1}^{\infty} a_{n} .
$$

Theorem 33. Let $\left\{\mathbf{e}_{n}\right\}_{n=1}^{\infty}$ be an orthonormal sequence in a real Hilbert space $\mathbb{V}$. Let $\left\{w_{n}\right\}_{n=1}^{\infty}$ be a sequence in $\mathbb{R}$ such that $0<$ $w_{n}<s$ for all $n$. For any sequence $\left\{r_{n}\right\}_{n=1}^{\infty}$ in $\mathbb{R}$, the following are equivalent:

(i) The series

$$
\begin{aligned}
& r_{1} \otimes w_{1} \mathbf{e}_{1} \oplus r_{2} \otimes w_{2} \mathbf{e}_{2} \oplus \cdots \oplus r_{n} \otimes w_{n} \mathbf{e}_{n} \oplus \cdots \\
& \text { converges to an element } \mathbf{x} \in \mathbb{V}_{s} .
\end{aligned}
$$

(ii) The series $\sum_{n=1}^{\infty}\left(r_{n} \otimes w_{n}\right)^{2} / s$ converges to $x \in \mathbb{R}$ with $|x|<s$.

Note that parentheses are not necessary in the formula in (i) above by Lemma 31.

Proof. (i) $\Rightarrow$ (ii). Put

$$
\begin{aligned}
& \mathbf{x}_{n}=r_{1} \otimes w_{1} \mathbf{e}_{1} \oplus \cdots \oplus r_{n} \otimes w_{n} \mathbf{e}_{n} \\
& \mathbf{x}=r_{1} \otimes w_{1} \mathbf{e}_{1} \oplus r_{2} \otimes w_{2} \mathbf{e}_{2} \oplus \cdots \oplus r_{n} \otimes w_{n} \mathbf{e}_{n} \oplus \cdots .
\end{aligned}
$$


From Lemma 15, it follows that

$$
\begin{aligned}
\frac{\left\|\mathbf{x}_{n}\right\|^{2}}{s} & =\frac{\left\|r_{1} \otimes w_{1} \mathbf{e}_{1}\right\|^{2}}{s} \oplus \cdots \oplus \frac{\left\|r_{n} \otimes w_{n} \mathbf{e}_{n}\right\|^{2}}{s} \\
& =\frac{\left(r_{1} \otimes w_{1}\right)^{2}}{s} \oplus \cdots \oplus \frac{\left(r_{n} \otimes w_{n}\right)^{2}}{s} .
\end{aligned}
$$

By the assumption, we have $h\left(\mathbf{x}, \mathbf{x}_{n}\right) \rightarrow 0$. It follows from Lemma 20(i) and Lemma 14(iii) that $\left\|\mathbf{x}_{n}\right\| \rightarrow\|\mathbf{x}\|$. Thus we have

$$
\lim _{n \rightarrow \infty} \sum_{j=1}^{n} \frac{\left(r_{j} \otimes w_{j}\right)^{2}}{s}=\frac{\|\mathbf{x}\|^{2}}{s}<s .
$$

(ii) $\Rightarrow$ (i). Suppose $x=\sum_{n=1}^{\infty}\left(r_{n} \otimes w_{n}\right)^{2} / s<s$. We put

$$
\begin{aligned}
& \mathbf{x}_{n}=r_{1} \otimes w_{1} \mathbf{e}_{1} \oplus \cdots \oplus r_{n} \otimes w_{n} \mathbf{e}_{n}, \\
& c_{n}=\sum_{j=1}^{n} \frac{\left(r_{j} \otimes w_{j}\right)^{2}}{s} .
\end{aligned}
$$

By the assumption, for any $\varepsilon>0$, there exists a number $n_{0}$ such that

$$
n \geq n_{0} \Longrightarrow 0 \leq x \ominus c_{n}<\varepsilon
$$

The last inequality implies that $\left(x-c_{n}\right) /\left(1-\left(1 / s^{2}\right) x c_{n}\right)<\varepsilon$. For $n_{0} \leq m<n$, by Lemma 15 ,

$$
\begin{aligned}
d\left(\mathbf{x}_{n}, \mathbf{x}_{m}\right)^{2} & =\left\|\ominus \mathbf{x}_{m} \oplus \mathbf{x}_{n}\right\|^{2} \\
& =\left\|r_{m+1} \otimes w_{m+1} \mathbf{e}_{m+1} \oplus \cdots \oplus r_{n} \otimes w_{n} \mathbf{e}_{n}\right\|^{2} \\
& =c_{n} \ominus c_{m}=\frac{c_{n}-c_{m}}{1-\left(1 / s^{2}\right) c_{n} c_{m}} \\
& \leq \frac{x-c_{m}}{1-\left(1 / s^{2}\right) x c_{m}}<\varepsilon .
\end{aligned}
$$

Note that the strict inequality $x<s$ is crucial in the argument above. This implies that $\left\{\mathbf{x}_{n}\right\}_{n}$ is a Cauchy sequence with respect to the metric $h$ by Lemma 20(ii). Since $\left(\mathbb{V}_{s}, h\right)$ is complete by Theorem 26 , there exists a unique element $\mathbf{x} \in \mathbb{V}_{s}$ such that $h\left(\mathbf{x}, \mathbf{x}_{n}\right) \rightarrow 0$. This completes the proof.

Example 34. Consider the sequence $\left\{a_{n}\right\}_{n=1}^{\infty}$ in $\mathbb{R}$ defined by $a_{n}=1 / 2 n$. For $s=1$, it is easy to see that

$$
x_{n}=a_{1} \oplus \cdots \oplus a_{n}=1-\frac{1}{n+1} \quad(n=1,2, \ldots) .
$$

Put $r_{n}=\tanh ^{-1}(1 / \sqrt{2 n}) / \tanh ^{-1}(1 / 2)$. Then, we have $r_{n} \otimes$ $(1 / 2)=\tanh \left(r_{n} \tanh ^{-1}(1 / 2)\right)=1 / \sqrt{2 n}$. It follows that

$$
\sum_{j=1}^{n}\left(r_{j} \otimes \frac{1}{2}\right)^{2}=\sum_{j=1}^{n} \frac{1}{2 j}=1-\frac{1}{n+1},
$$

which does not converge to an element $x \in \mathbb{R}$ with $|x|<$ 1. This example can be considered as a counterpart in the Möbius gyrovector space to the series $\sum_{n=1}^{\infty}(1 / 2 n)$.
Theorem 35. Let $\left\{\mathbf{e}_{n}\right\}_{n=1}^{\infty}$ be a complete orthonormal sequence in a real Hilbert space $\mathbb{V}$. Let $\left\{w_{n}\right\}_{n=1}^{\infty}$ be a sequence in $\mathbb{R}$ such that $0<w_{n}<s$ for all $n$. Then, for any $\mathbf{x} \in \mathbb{V}_{s}$, we have the orthogonal gyroexpansion

$$
\mathbf{x}=r_{1} \otimes w_{1} \mathbf{e}_{1} \oplus r_{2} \otimes w_{2} \mathbf{e}_{2} \oplus \cdots \oplus r_{n} \otimes w_{n} \mathbf{e}_{n} \oplus \cdots
$$

where the sequence of gyrocoefficients $\left\{r_{n}\right\}_{n=1}^{\infty}$ is determined by the following equations:

$$
\begin{aligned}
& x_{n}=\mathbf{x} \cdot \mathbf{e}_{n}, \\
& \mathbf{x}_{n}^{(1)}=\sum_{j=1}^{n} x_{j} \mathbf{e}_{j}, \\
& \mathbf{x}_{n}^{(2)}=\sum_{j=n+1}^{\infty} x_{j} \mathbf{e}_{j} \\
& \mathbf{u}_{j}=\mu_{j-1}^{(2)} \cdots \mu_{1}^{(2)} x_{j} \mathbf{e}_{j} \quad(j=2,3, \ldots) \\
& \mathbf{u}_{1}=x_{1} \mathbf{e}_{1}=\mathbf{x}_{1}^{(1)} \\
& \mathbf{v}_{j}=\mu_{j-1}^{(2)} \cdots \mu_{1}^{(2)} \mathbf{x}_{j}^{(2)} \quad(j=2,3, \ldots) \\
& \mathbf{v}_{1}=\mathbf{x}_{1}^{(2)} \\
& \mu_{j}^{(1)} \\
& r_{j}=\frac{\tanh ^{-1}\left(\mu_{j}^{(1)} \mu_{j-1}^{(2)} \cdots \mu_{1}^{(2)} x_{j} / s\right)}{\tanh ^{-1}\left(w_{j} / s\right)} \\
& =\frac{\left\|\mathbf{u}_{j}\right\|^{2}+\left\|\mathbf{v}_{j}\right\|^{2}+s^{2}-\sqrt{\left(\left\|\mathbf{u}_{j}\right\|^{2}+\left\|\mathbf{v}_{j}\right\|^{2}+s^{2}\right)^{2}-4 s^{2}\left\|\mathbf{u}_{j}\right\|^{2}}}{2\left\|\mathbf{u}_{j}\right\|^{2}} \\
& \mu_{j}^{(2)} \\
& =\frac{\left\|\mathbf{u}_{j}\right\|^{2}+\left\|\mathbf{v}_{j}\right\|^{2}-s^{2}+\sqrt{\left(\left\|\mathbf{u}_{j}\right\|^{2}+\left\|\mathbf{v}_{j}\right\|^{2}+s^{2}\right)^{2}-4 s^{2}\left\|\mathbf{u}_{j}\right\|^{2}}}{2\left\|\mathbf{v}_{j}\right\|^{2}}
\end{aligned}
$$

for all $j, n=1,2, \ldots$. If $x_{j}=0$, then we do not define $\mu_{j}^{(1)}$ but define as $r_{j}=0$ and continue the procedure. If $\mathbf{v}_{n}=\mathbf{0}$, then we do not define $\mu_{n}^{(2)}$ but define as $r_{j}=0$ for all $j \geq n+1$ and finish the procedure.

Proof. It is not difficult to see that we may assume $s=1$. It is obvious that the series $\sum_{n=1}^{\infty} x_{n} \mathbf{e}_{n}$ converges to $\mathbf{x}$ in the norm topology and that

$$
\mathbf{x}=\mathbf{x}_{n}^{(1)}+\mathbf{x}_{n}^{(2)}
$$

is the orthogonal decomposition with respect to the closed linear subspace generated by $\left\{\mathbf{e}_{1}, \ldots, \mathbf{e}_{n}\right\}$. Let

$$
\mathbf{x}=\mathbf{y}_{n} \oplus \mathbf{z}_{n}, \quad \mathbf{y}_{n} \in M_{n}, \quad \mathbf{z}_{n} \in M_{n}{ }^{\perp} \cap \mathbb{V}_{1}
$$


be the orthogonal gyrodecomposition with respect to $M_{n}$ by Theorem 27 and [8, Theorem 4.2], where $M_{n}$ is an $h$ closed gyrovector subspace generated by $\left\{w_{1} \mathbf{e}_{1}, \ldots, w_{n} \mathbf{e}_{n}\right\}$. Then, $\mathbf{y}_{n}, \mathbf{z}_{n}$ are given by the equations

$$
\begin{aligned}
& \mathbf{y}_{n}=\lambda_{n}^{(1)} \mathbf{x}_{n}^{(1)}, \\
& \mathbf{z}_{n}=\lambda_{n}^{(2)} \mathbf{x}_{n}^{(2)},
\end{aligned}
$$

where

$$
\begin{aligned}
& \lambda_{n}^{(1)}=\frac{\|\mathbf{x}\|^{2}+1-\sqrt{\left(\|\mathbf{x}\|^{2}+1\right)^{2}-4\left\|\mathbf{x}_{n}^{(1)}\right\|^{2}}}{2\left\|\mathbf{x}_{n}^{(1)}\right\|^{2}} \\
& \lambda_{n}^{(2)}=\frac{\|\mathbf{x}\|^{2}-1+\sqrt{\left(\|\mathbf{x}\|^{2}+1\right)^{2}-4\left\|\mathbf{x}_{n}^{(1)}\right\|^{2}}}{2\left\|\mathbf{x}_{n}^{(2)}\right\|^{2}} .
\end{aligned}
$$

Since $\left\|\mathbf{x}_{n}^{(1)}-\mathbf{x}\right\| \rightarrow 0$

$$
\lambda_{n}^{(1)} \longrightarrow \frac{\|\mathbf{x}\|^{2}+1-\sqrt{\left(\|\mathbf{x}\|^{2}+1\right)^{2}-4\|\mathbf{x}\|^{2}}}{2\|\mathbf{x}\|^{2}}=1,
$$

so that we have

$$
\mathbf{y}_{n}=\lambda_{n}^{(1)} \mathbf{x}_{n}^{(1)} \longrightarrow \mathbf{x}
$$

in the norm topology, which implies that $h\left(\mathbf{y}_{n}, \mathbf{x}\right) \rightarrow 0$. tion

Next, we express $\mathbf{y}_{n}$ in the form of a gyrolinear combina-

$$
\mathbf{y}_{n}=r_{1} \otimes w_{1} \mathbf{e}_{1} \oplus r_{2} \otimes w_{2} \mathbf{e}_{2} \oplus \cdots \oplus r_{n} \otimes w_{n} \mathbf{e}_{n}
$$

and present a concrete procedure to seek the gyrocoefficients $r_{n}$.

For $n=1$, by using the above decomposition, we take $r_{1}=\tanh ^{-1} \lambda_{1}^{(1)} x_{1} / \tanh ^{-1} w_{1}$. It follows that

$$
\begin{aligned}
r_{1} \otimes w_{1} \mathbf{e}_{1} & =\tanh \left(r_{1} \tanh ^{-1}\left\|w_{1} \mathbf{e}_{1}\right\|\right) \frac{w_{1} \mathbf{e}_{1}}{\left\|w_{1} \mathbf{e}_{1}\right\|} \\
& =\lambda_{1}^{(1)} x_{1} \mathbf{e}_{1}=\lambda_{1}^{(1)} \mathbf{x}_{1}^{(1)}=\mathbf{y}_{1} \\
\mathbf{x} & =r_{1} \otimes w_{1} \mathbf{e}_{1} \oplus \mathbf{z}_{1} .
\end{aligned}
$$

Suppose that we proceed up to the $n$-th step and obtain the quantities, identities (110) for $j=1, \ldots, n$, and

$$
\mathbf{x}=r_{1} \otimes w_{1} \mathbf{e}_{1} \oplus \cdots \oplus r_{n} \otimes w_{n} \mathbf{e}_{n} \oplus \mu_{n}^{(2)} \mathbf{v}_{n} .
$$

Now,

$$
\mu_{n}^{(2)} \mathbf{v}_{n}=\mu_{n}^{(2)} \cdots \mu_{1}^{(2)} x_{n+1} \mathbf{e}_{n+1}+\mu_{n}^{(2)} \cdots \mu_{1}^{(2)} \sum_{j=n+2}^{\infty} x_{j} \mathbf{e}_{j}
$$

$$
=\mathbf{u}_{n+1}+\mathbf{v}_{n+1}
$$

is the orthogonal decomposition with respect to the finite dimensional linear subspace generated by $\left\{\mathbf{e}_{1}, \ldots, \mathbf{e}_{n+1}\right\}$. Let

$$
\mu_{n}^{(2)} \mathbf{v}_{n}=\mathbf{y}_{n+1}^{\prime} \oplus \mathbf{z}_{n+1}^{\prime} \text {, }
$$

$$
\mathbf{y}_{n+1}^{\prime} \in M_{n+1}, \mathbf{z}_{n+1}^{\prime} \in M_{n+1}^{\perp} \cap \mathbb{V}_{1}
$$

be the orthogonal gyrodecomposition with respect to $M_{n+1}$. Then, $\mathbf{y}_{n+1}^{\prime}, \mathbf{z}_{n+1}^{\prime}$ are given by the equations

$$
\begin{aligned}
& \mathbf{y}_{n+1}^{\prime}=\mu_{n+1}^{(1)} \mathbf{u}_{n+1}, \\
& \mathbf{z}_{n+1}^{\prime}=\mu_{n+1}^{(2)} \mathbf{v}_{n+1},
\end{aligned}
$$

where

$$
\begin{aligned}
& \mu_{n+1}^{(1)}=\frac{\left\|\mathbf{u}_{n+1}\right\|^{2}+\left\|\mathbf{v}_{n+1}\right\|^{2}+1-\sqrt{\left(\left\|\mathbf{u}_{n+1}\right\|^{2}+\left\|\mathbf{v}_{n+1}\right\|^{2}+1\right)^{2}-4\left\|\mathbf{u}_{n+1}\right\|^{2}}}{2\left\|\mathbf{u}_{n+1}\right\|^{2}} \\
& \mu_{n+1}^{(2)}=\frac{\left\|\mathbf{u}_{n+1}\right\|^{2}+\left\|\mathbf{v}_{n+1}\right\|^{2}-1+\sqrt{\left(\left\|\mathbf{u}_{n+1}\right\|^{2}+\left\|\mathbf{v}_{n+1}\right\|^{2}+1\right)^{2}-4\left\|\mathbf{u}_{n+1}\right\|^{2}}}{2\left\|\mathbf{v}_{n+1}\right\|^{2}} .
\end{aligned}
$$

By taking

$$
r_{n+1}=\frac{\tanh ^{-1}\left(\mu_{n+1}^{(1)} \mu_{n}^{(2)} \cdots \mu_{1}^{(2)} x_{n+1}\right)}{\tanh ^{-1} w_{n+1}},
$$

we have

$$
\begin{aligned}
\mathbf{x} & =r_{1} \otimes w_{1} \mathbf{e}_{1} \oplus \cdots \oplus r_{n} \otimes w_{n} \mathbf{e}_{n} \oplus\left(\mathbf{y}_{n+1}^{\prime} \oplus \mathbf{z}_{n+1}^{\prime}\right) \\
& =r_{1} \otimes w_{1} \mathbf{e}_{1} \oplus \cdots \oplus r_{n} \otimes w_{n} \mathbf{e}_{n}
\end{aligned}
$$

$$
\begin{aligned}
& \oplus\left(\mu_{n+1}^{(1)} \mathbf{u}_{n+1} \oplus \mu_{n+1}^{(2)} \mathbf{v}_{n+1}\right) \\
= & r_{1} \otimes w_{1} \mathbf{e}_{1} \oplus \cdots \oplus r_{n} \otimes w_{n} \mathbf{e}_{n} \oplus r_{n+1} \otimes w_{n+1} \mathbf{e}_{n+1} \\
& \oplus \mu_{n+1}^{(2)} \mathbf{v}_{n+1} .
\end{aligned}
$$

Thus, we can inductively take a sequence $\left\{r_{n}\right\}_{n=1}^{\infty}$ by the procedure above. 
Finally, from the uniqueness of the orthogonal gyrodecomposition with respect to the $h$-closed gyrovector subspace $M_{n}$, it follows that

$$
\mathbf{y}_{n}=r_{1} \otimes w_{1} \mathbf{e}_{1} \oplus \cdots \oplus r_{n} \otimes w_{n} \mathbf{e}_{n}
$$

and the series converges as follows:

$$
\mathbf{x}=r_{1} \otimes w_{1} \mathbf{e}_{1} \oplus \cdots \oplus r_{n} \otimes w_{n} \mathbf{e}_{n} \oplus \cdots
$$

This completes the proof.

Theorem 36. Let $\left\{\mathbf{e}_{n}\right\}_{n=1}^{\infty}$ be an orthonormal sequence in a real Hilbert space $\mathbb{V}$. Let $\left\{w_{n}\right\}_{n=1}^{\infty}$ be a sequence in $\mathbb{R}$ such that $0<$ $w_{n}<s$ for all $n$. Then the following are equivalent:

(i) $\left\{\mathbf{e}_{n}\right\}_{n=1}^{\infty}$ is complete.

(ii) The $h$-closed gyrovector subspace generated by $\left\{w_{n} \mathbf{e}_{n}\right\}_{n=1}^{\infty}$ coincides with $\mathbb{V}_{s}$.

(iii) $\|\mathbf{x}\|^{2}=\sum_{n=1}^{\infty}\left(r_{n} \otimes w_{n}\right)^{2} / s$ for all $\mathbf{x} \in \mathbb{V}_{s}$,

where $\left\{r_{n}\right\}_{n=1}^{\infty}$ is the sequence determined by identities (110).

Proof. It is easy to deduce implications (i) $\Rightarrow$ (ii) and (i) $\Rightarrow$ (iii) from the previous theorem.

(iii) $\Rightarrow$ (i) Suppose that there exists an element $\mathbf{x} \neq \mathbf{0}$ such that $\mathbf{x} \cdot \mathbf{e}_{n}=0$ for all $n$. By multiplying nonzero scalar, we may assume that $\mathbf{x} \in \mathbb{V}_{s}$. Then we have $x_{n}=0$, hence $r_{n}=0$ for all $n$. Thus (iii) is violated.

(ii) $\Rightarrow$ (i) Suppose that there exists an element $\mathbf{x}$ such that $\mathbf{x} \cdot \mathbf{e}_{n}=0$ for all $n$. We may assume that $\mathbf{x} \in \mathbb{V}_{s}$. Then $\{\mathbf{x}\}^{\perp} \cap \mathbb{V}_{s}$ is an $h$-closed gyrovector subspace by Lemma 22 . Since it contains $w_{n} \mathbf{e}_{n}$ for all $n$, it coincides with $\mathbb{V}_{s}$ by the assumption. Therefore, we have $\mathbf{x} \in\{\mathbf{x}\}^{\perp} \cap \mathbb{V}_{s}$, which implies that $\mathbf{x}=\mathbf{0}$. This completes the proof.

\section{Conflicts of Interest}

The author declares that there are no conflicts of interest regarding the publication of this article.

\section{Acknowledgments}

The author would like to thank Professor Michio Seto for bringing information on literatures $[11,12]$.

\section{References}

[1] A. A. Ungar, "Thomas rotation and the parametrization of the Lorentz transformation group," Foundations of Physics Letters, vol. 1, no. 1, pp. 57-89, 1988.

[2] A. A. Ungar, "Extension of the unit disk gyrogroup into the unit ball of any real inner product space," Journal of Mathematical Analysis and Applications, vol. 202, no. 3, pp. 1040-1057, 1996.

[3] A. A. Ungar, "Group-like structure underlying the unit ball in real inner product spaces," Results in Mathematics, vol. 18, no. 3-4, pp. 355-364, 1990.

[4] A. A. Ungar, Analytic hyperbolic geometry and Albert Einstein's special theory of relativity, World Scientific Publishing Co. Pte. Ltd., Toh Tuck Link, Singapore, 2008.
[5] A. A. Ungar, Analytic Hyperbolic Geometry, World Scientific Publishing Co. Pte. Ltd., Hackensack, NJ, USA, 2005.

[6] T. Abe and O. Hatori, "On a characterization of commutativity for $C^{*}$-algebras via gyrogroup operations," Periodica Mathematica Hungarica, vol. 72, no. 2, pp. 248-251, 2016.

[7] T. Abe and O. Hatori, "Generalized gyrovector spaces and a Mazur-Ulam theorem," Publicationes Mathematicae Debrecen, vol. 87, no. 3-4, pp. 393-413, 2015.

[8] T. Abe and K. Watanabe, "Finitely generated gyrovector subspaces and orthogonal gyrodecomposition in the Möbius gyrovector space," Journal of Mathematical Analysis and Applications, vol. 449, no. 1, pp. 77-90, 2017.

[9] O. Hatori, "Examples and applications of generalized gyrovector spaces," Results in Mathematics, vol. 71, no. 1-2, pp. 295-317, 2017.

[10] K. Watanabe, "A confirmation by hand calculation that the Möbius ball is a gyrovector space," Nihonkai Mathematical Journal, vol. 27, no. 1-2, pp. 99-115, 2016.

[11] K. Goebel and S. Reich, Uniform Convexity, Hyperbolic Geometry and Nonexpansive Mappings, vol. 83 of Monographs and Textbooks in Pure and Applied Mathematics, Marcel Dekker, New York, NY, USA, 1984.

[12] M. Bacák, Convex Analysis and Optimization in Hadamard Spaces, vol. 22 of De Gruyter Series in Nonlinear Analysis and Applications, Walter de Gruyter, Berlin, Germany, 2014. 


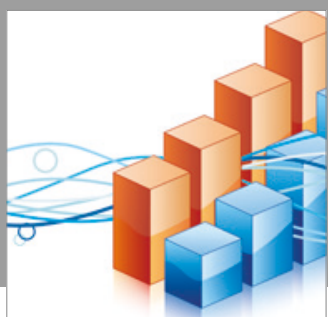

Advances in

Operations Research

vatersals

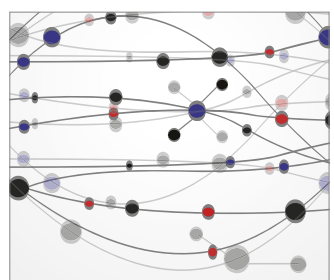

\section{The Scientific} World Journal
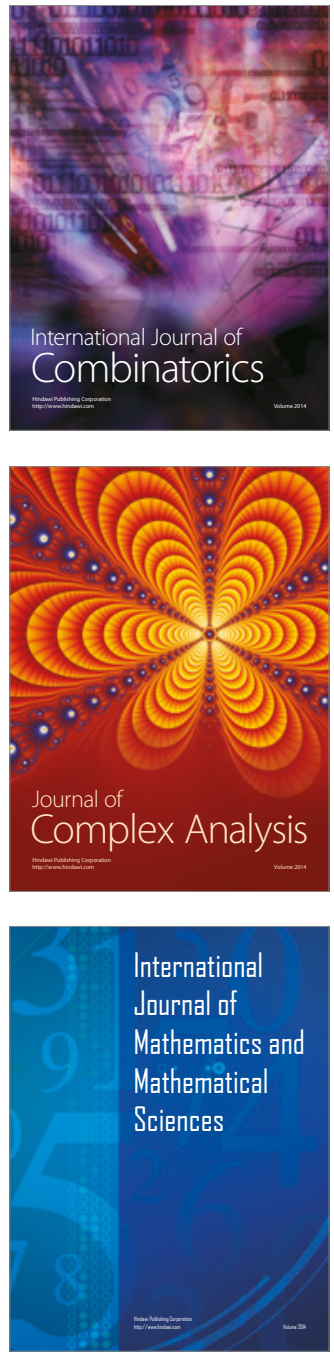
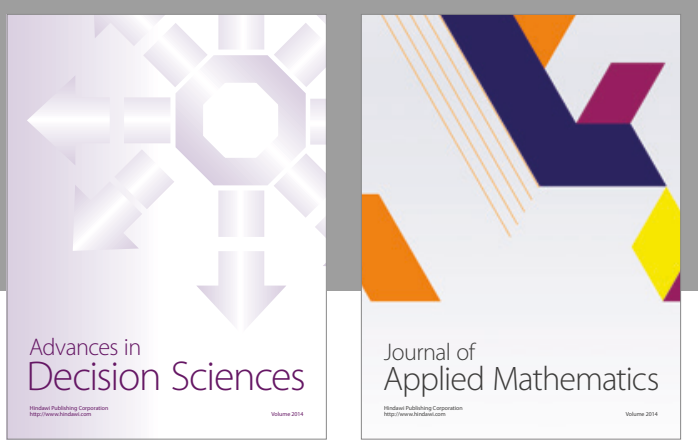

Algebra

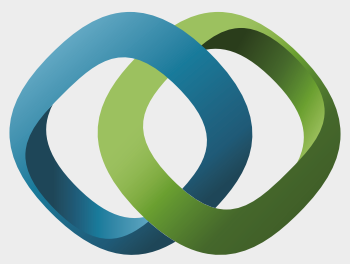

\section{Hindawi}

Submit your manuscripts at

https://www.hindawi.com
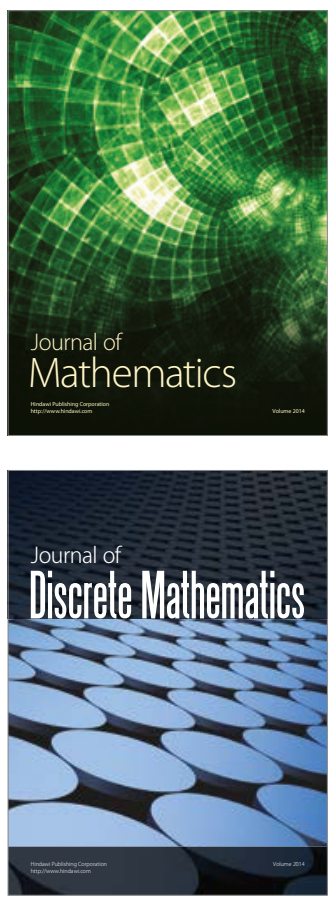

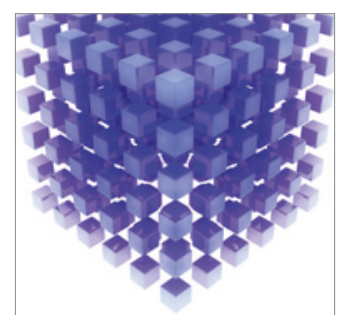

Mathematical Problems in Engineering
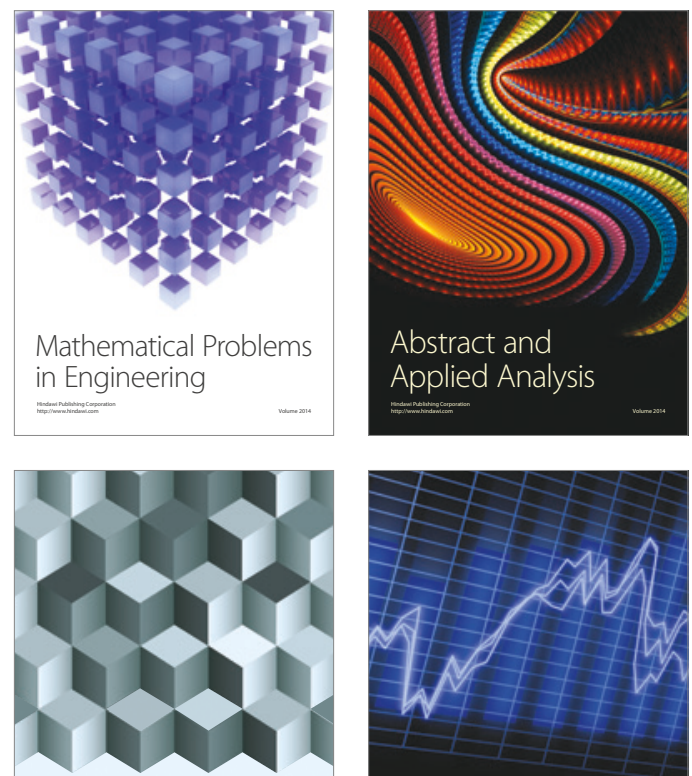

Journal of

Function Spaces

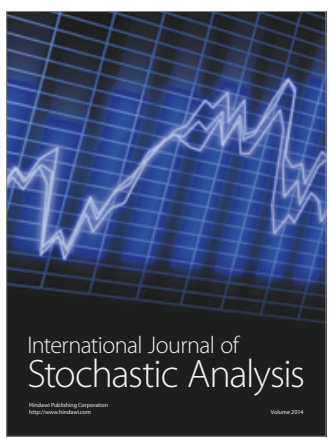

Probability and Statistics
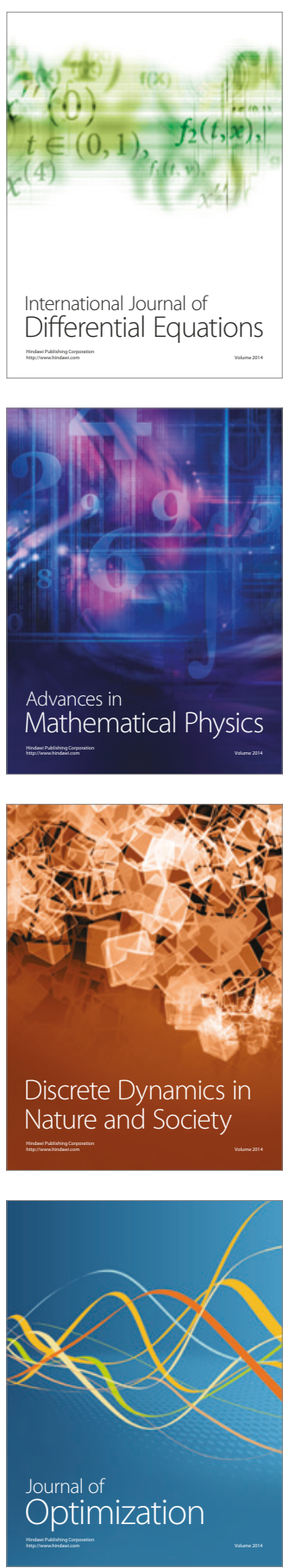